Research Article

\title{
A QFD-Based Quality and Capability Design Method for Transboundary Services
}

\author{
Chao Ma, ${ }^{1}$ Wei Dong Liu, ${ }^{2}$ Zhi Ying Tu, ${ }^{2}$ Zhong Jie Wang $\mathbb{D}^{2},{ }^{2}$ and Xiao Fei $X u^{2}$ \\ ${ }^{1}$ School of Software and Microelectronics, Harbin University of Science and Technology, Harbin, China \\ ${ }^{2}$ School of Computer Science and Technology, Harbin Institute of Technology, Harbin, China \\ Correspondence should be addressed to Zhong Jie Wang; rainy@hit.edu.cn
}

Received 18 October 2019; Revised 12 December 2019; Accepted 1 February 2020; Published 24 February 2020

Academic Editor: Ricardo Aguilar-Lopez

Copyright (c) 2020 Chao Ma et al. This is an open access article distributed under the Creative Commons Attribution License, which permits unrestricted use, distribution, and reproduction in any medium, provided the original work is properly cited.

The "transboundary", an emerging phenomenon in the Internet service ecosystem, is leading to the flourishing of innovative services. A transboundary service incorporates services, resources, and technologies from multiple domains into its business to create a particular competitive advantage and unique user experiences. It is difficult to comprehensively consider all the constraints from multiple domains to precisely design the nonfunctional characteristics of transboundary services, such as quality attributes and capability attributes. We propose a two-phase quality design method for transboundary services called value quality deployment-quality capability deployment (VQD-QCD) based on quality function deployment (QFD). Given the restrictions of transboundary services, VQD-QCD translates the value expectations of multiple stakeholders into an optimal configuration for global quality parameters (GQPs), local quality parameters, and capability parameters. Details of VQD are illustrated. Considering the inherent vagueness and uncertainty of relationships between value expectations and GQPs, and among GQPs, fuzzy least absolute regression and fuzzy nonlinear programming methods are incorporated into QFD to identify the quantitative relations between value indicators and GQPs, and among GQPs, and obtain an optimal configuration scheme for GQPs. Usability of the proposed method is validated through a case study on the "DiDi mobile transportation service", which is a representative transboundary service in China. Compared with the current method, which is inaccurate and inefficient because its translation between value expectations and relevant quality and capability parameters is artificial and subjective, the proposed method integrates fuzzy least absolute regression and fuzzy nonlinear programming methods into QFD, which facilitate transboundary service designers to precisely and efficiently design the quality and capability characteristics of innovative services in the manner of semiautomatisation, which promotes the innovative design of transboundary services.

\section{Introduction}

Recently, numerous innovative services have appeared with an emerging phenomenon: the "transboundary" (also called "crossover"), for example, the Didi (https://www.didiglobal. com) service that belongs to the online taxi-hailing service field, Fliggy (https://www.fliggy.com) service that belongs to the online travel service field, and Nongcuntaobao (https:// cun.taobao.com) service that belongs to the online retail service field. This type of transboundary service invents a brand new business model by incorporating services, resources, or technologies from multiple domains into its business to create a particular competitive advantage for itself and unique user experiences for its customers. The essence of a transboundary service is the deep convergence of multiple independent services from different industries, different organisations, and different value chains [1]. The Internet of Services (IoS) [2] and Big Service [3] are two similar terms that have attracted wide attention in recent years.

The design of transboundary services requires two phases: functional design and quality/capability design. In terms of functional design, designers incorporate specific design tools and their domain knowledge and personal experiences. For example, using the business process model and notation as the modelling language, designers can accurately delineate the transboundary collaboration process among multiple participants (i.e., stakeholders and 
customers) of a transboundary service, as shown on the lefthand side of Figure 1. This collaboration process consists of a set of activities, a set of resources, and the bindings between resources and activities.

In terms of quality/capability design, however, the "transboundary" makes it difficult to comprehensively consider all the constraints from multiple domains to precisely design the nonfunctional characteristics of transboundary services, as shown on the right-hand side of Figure 1. These nonfunctional characteristics consist of global quality parameters (GQPs) attached to the transboundary service, local quality parameters (LQPs) attached to the activities, and capability parameters (CPs) attached to the resources. Therefore, in this paper, we focus on the phase of quality and capability design.

Compared with traditional service design in which design objectives are solely determined by one service provider, the design objectives of a transboundary service originate from the convergence of multiple stakeholders of the service. These stakeholders are attached to different business domains, have different value expectations, and own different service capabilities. There might be implicit conflicts or correlations among these nonfunctional perspectives, and the design of a transboundary service has to carefully consider all these perspectives and seek a global trade-off between them so that the stakeholders can collaborate effectively to reach the shared goal of the transboundary service.

In our opinion, the design process of a transboundary service is two-fold: "top-down," that is, transforming value expectations of all stakeholders of the service into a quality/ capability configuration scheme for the service functionalities, activities, and resources of the transboundary service (i.e., only if the service functionalities, activities, and resources reach such levels of quality and capability can the value expectations of all stakeholders be fully achieved); and "bottom-up," that is, if the capabilities that could be actually offered by these stakeholders are too limited to support the achievement of value expectations, the optimal quality/capability configuration scheme cannot be obtained, so an adjustment of the original value expectations has to be made. However, before engaging in an adjustment in a bottom-up manner, there are many complex top-down details that need to be understood, which this paper tries to do.

The "top-down" design challenge is how to transform the value expectations of various stakeholders into a configuration scheme of multiple relevant quality/capability parameters accurately and efficiently. However, because there is no appropriate method, current transboundary service designers conduct this transformation artificially, which makes it difficult for designers to obtain an accurate quality/ capability configuration scheme efficiently. Therefore, to meet the "top-down" design challenge of a transboundary service, in this paper, we propose a quality and capability design method for transboundary services to help designers to identify the optimal configuration scheme by incorporating quality function deployment (QFD) [4] and elaborate on considerations regarding the complex correlations between value expectations and the available capabilities of multiple stakeholders of a transboundary service.
QFD is a customer-driven product design method. Based on the philosophy of the system engineering theory, it translates customer needs for a product layer by layer into the production requirements and fulfils customer needs in the product design stage. Many early studies [5-9] are incomplete in the sense that they either do not contain all the important elements of QFD or do not handle well most information generated from human beings' perceptions and linguistic assessments that are quite subjective and vague. Chan et al. proposed a systematic approach to quality function deployment with a nine-step model for building the house of quality (HoQ) [10], which is a systematic and operational approach to the QFD process. However, its 1-to9 scale that could help to unify the various measurements in QFD is not suitable for the optimal quality and capability design of transboundary services, and its nine-step model also cannot fully satisfy the optimal quality and capability design needs of transboundary services.

By incorporating QFD in the requirement development process (RDP) of software systems, QFD can facilitate the design of software systems by transforming user requirements into critical-to-system functions and then into functional requirements [11]. QFD-based RDP proposed for the design of software systems is not suitable for the design of transboundary services, and it also does not handle well most subjective and vague information generated from human beings' perceptions and linguistic assessments. In our previous work, a QFD-based service quality design method (SQFD) was presented, which consecutively transforms user expectations into the quality parameters of the service requirement model (SRM), service behaviour and capability model (SBCM), and service execution model to eliminate the gap between user expectations and service quality that customers perceive [12]. However, SQFD is applied only for the quality design of a service that is offered by a single provider and cannot manage the challenge of the transboundary convergence of multiple existing services offered by different service providers.

In this paper, we propose a two-phase model for the optimal quality and capability design of transboundary services called value quality deployment-quality capability deployment (VQD-QCD). As shown on the right-hand side of Figure 1, in the first phase, VQD transforms the value expectations of multiple stakeholders from different domains into a configuration scheme for GQPs of transboundary services. In the second phase, QCD transforms the configuration scheme for GQPs into a configuration scheme for LQPs and a configuration scheme for CPs. Methods that include fuzzy least absolute regression (FLAR) [13] and fuzzy nonlinear programming [14] are incorporated in the HoQ of classical QFD so that the optimal configuration schemes for GQPs, LQPs, and CPs are generated in the topdown manner. VQD-QCD can facilitate transboundary service designers to precisely design quality/capability characteristics of innovative services. It will become a popular design method for innovative services because it provides effective means for service innovation.

The main contributions of this paper are summarised as follows: 


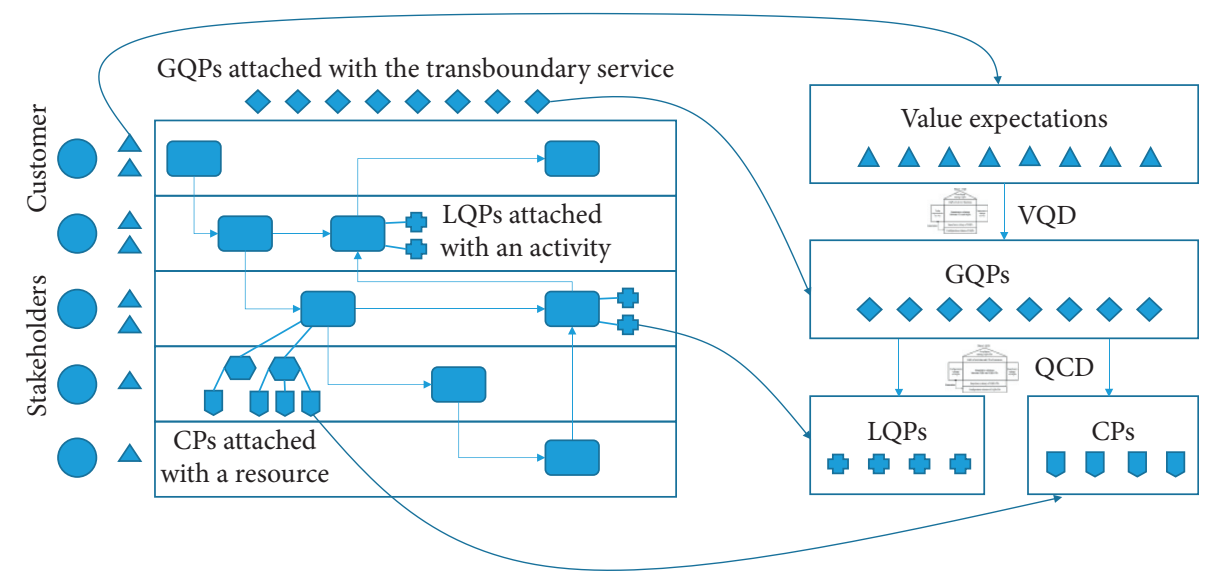

FIGURE 1: Design of transboundary services.

(1) We present VQD-QCD for the nonfunctional design of transboundary services by elaborating on the considerations of value expectation conflicts, quality attribute correlations, and constraints of the actual service capabilities of different service providers from different business domains.

(2) We apply a two-phase design process in VQD-QCD to seek the trade-off between high-level value expectations and real-world service capabilities. The optimal configuration scheme for GQPs, LQPs, and CPs is obtained in the top-down manner.

(3) Considering the inherent vagueness and uncertainty of relationships among value indicators (VIs) and GQPs of transboundary services, we incorporate FLAR and fuzzy nonlinear programming methods into VQD to identify quantitative relations between VIs and GQPs and the correlations among GQPs.

The remainder of this paper is organised as follows: In Section 2, we present the two-phase model VQD-QCD. In Section 3, we explain the detailed design process of VQD. In Section 4, we demonstrate the use of the FLAR method to identify the quantitative relations between VIs and GQPs and the correlations among GQPs. In Section 5, we explain the use of fuzzy nonlinear programming model for the optimised design of the configuration scheme for GQPs. In Section 6, we present a case study on the "DiDi transportation service (which is referred to as DiDi hereafter) to validate the usability of the proposed method. In the final section, we present the conclusion and future work.

\section{Overview of VQD-QCD}

In Table 1, we compare our proposed VQD-QCD and QFD [10], QFD-based RDP [11], and SQFD [12]. In terms of the design targets, QFD is proposed for the design of products and QFD-based RDP is proposed for the design of manufacturing information systems, whereas SQFD and VQD-QCD are for the design of services. In terms of the core method, all the methods are based on the HoQ. In terms of handling subjective and vague information generated from human beings' perceptions and linguistic assessments, symmetric triangular fuzzy numbers (STFNs) are used to capture the vagueness in QFD and FLAR is used to capture the vagueness in VQD-QCD, but the related methods are not explained in detail for QFD-based RDP and SQFD. Additionally, SQFD is applied only for the quality design of a service that is offered by a single provider and cannot handle well the value expectation conflicts, quality attribute correlations, and constraints of the actual service capabilities of different service providers from different business domains. In conclusion, our VQDQCD is focused on transboundary services that have multiple stakeholders from multiple domains, and the other three methods cannot manage the challenges posed by the "transboundary".

The two-phase framework of VQD-QCD is shown in Figure 2. The VQD phase supports the design between value expectations of multiple stakeholders and the GQPs of service functions, and the QCD phase supports the design between the GQPs and LQPs of fine-grained service activities and CPs of service resources. VQD-QCD is the topdown generative design of a quality/capability configuration scheme.

The input of VQD-QCD includes the following: (1) the functional design of a transboundary service, including a business process that consists of a set of activities, a set of resources with specific capabilities, and the bindings between resources and activities; (2) value expectations of multiple stakeholders who participate in the transboundary service by offering specific resources and are responsible for executing specific activities, including a set of VIs and constraints on them; and (3) the available quality levels of activities and capability of available resources that each stakeholder can offer to the transboundary service. The output of VQD-QCD includes the following: (1) configuration scheme for GQPs and (2) configuration scheme for LQPs and CPs.

In the VQD phase, the value expectations on VIs are the input on the left-hand side of the HoQ, and the importance rating of each VI is calculated. At the centre of the HoQ, quantitative relations between the VIs and GQPs of service functions are identified, and how and to what degree the realisation of each VI is affected by GQPs. The importance 
TABLE 1: Comparison of the QCD-VQD with QFD, QFD-based RDP, and SQFD.

\begin{tabular}{lcccc}
\hline $\begin{array}{l}\text { Related } \\
\text { methods }\end{array}$ & Targets to be designed & Phase 1 & \multicolumn{2}{c}{ Deployment process } \\
QFD & Products & VOCB & Product CTQ & Part CTQ \\
\hline $\begin{array}{l}\text { QFD-based } \\
\text { RDP }\end{array}$ & Manufacturing information systems & URs & $\begin{array}{c}\text { Critical-to-system } \\
\text { functions }\end{array}$ & Functional requirements \\
\hline SQFD & Services with one single provider & UEs & QPs of SRM & QPs of SBCM \\
\hline VQD-QCD & Transboundary services with multiple stakeholders from & VEs & GQPs & LQPs/CPs \\
\hline
\end{tabular}

VOCB: voice of customers and business; CTQ: critical to quality; URs: user requirements; UEs: user expectations; QPs: quality parameters; VEs: value expectations.

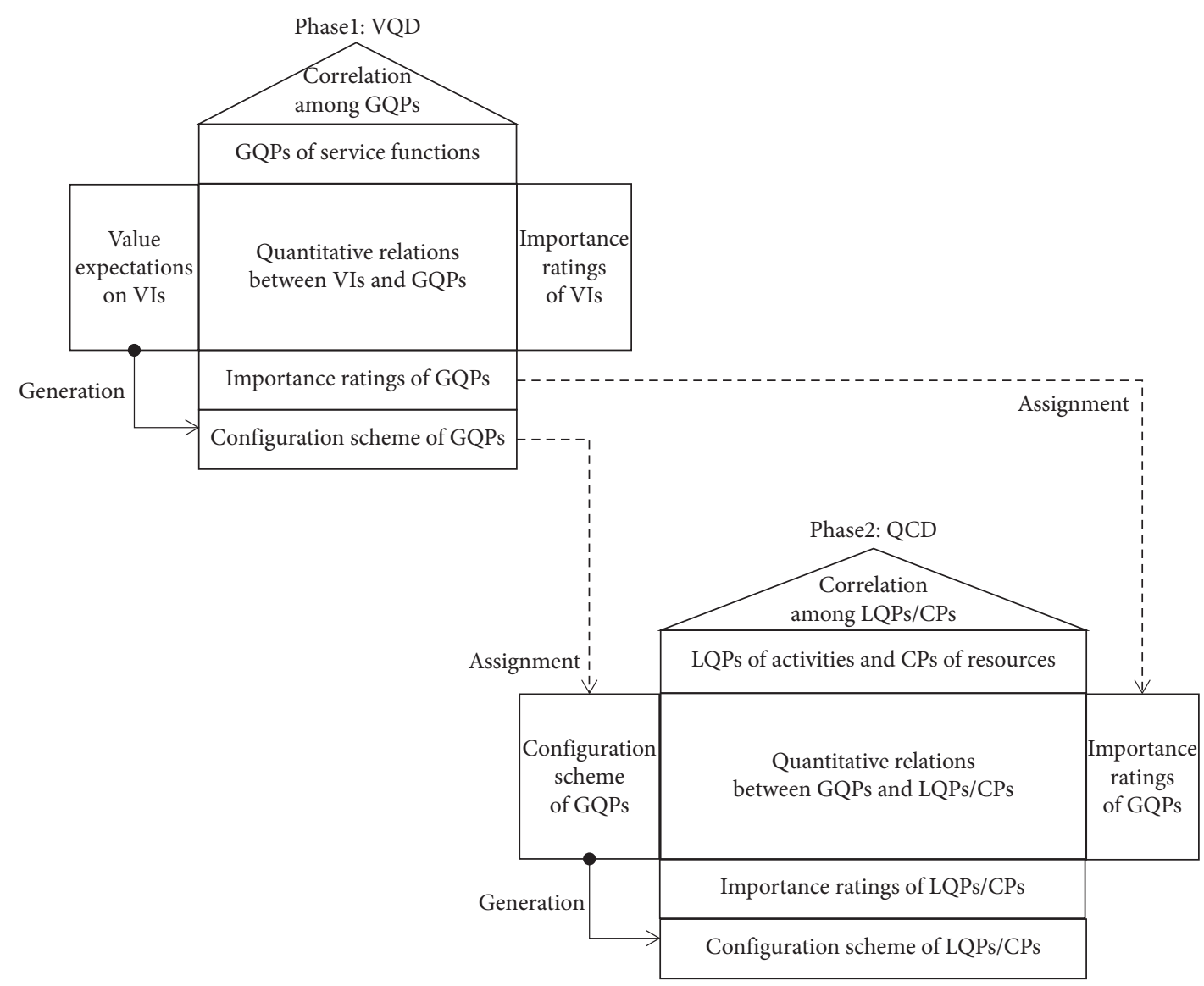

$\longrightarrow$ VQD/QCD: top-down generative design of configuration scheme

$\rightarrow-\rightarrow$ VQD $\rightarrow$ QCD: assign the output of VQD to the input of QCD

Figure 2: Two-phase framework of VQD-QCD.

ratings of the GQPs are calculated and listed in the penultimate row of the HoQ, and the value range of each GQP is calculated using an optimisation algorithm and listed at the bottom of the HoQ as the output of VQD, that is, the configuration scheme for GQPs. This is the top-down generative design of the GQP configuration scheme.

QCD is similar to VQD, but the objective is to design the configuration scheme for LQPs and CPs. First, the configuration scheme and importance ratings of GQPs, which are exactly the output of VQD, are used as the input on the left-hand side of the HoQ. Second, the quantitative relations between the "GQPs of service functions" and "LQPs of activities and CPs of resources" are identified and filled in at the centre of the HoQ. Next, the importance ratings of LQPs/ $\mathrm{CPs}$ are calculated, and then the configuration scheme for LQPs/CPs is obtained as the output of QCD using an optimisation algorithm.

The top-down design stages are conducted until reasonable configuration schemes (for all GQPs, LQPs, and $\mathrm{CPs}$ ) that are restricted by both value expectations and 
available real-world quality/capabilities are obtained. We use DiDi as a transboundary service example to illustrate the design process mentioned above. In $\mathrm{DiDi}$, there are many participants, such as the DiDi app provider, map service provider, taxi drivers, and individual travellers. Each participant has its own value expectations on VIs such as "Market Share," "Daily Order Volume," "Average Daily Income", and "Favourable Rate." For example, the app provider proposes the value expectation "Market Share is larger than or equal to $70 \%$," the community of taxi drivers has the value expectation "Average Daily Income of Drivers is larger than 300 RMB Yuan," and the community of travellers has the value expectation "Favourable Rate is larger than or equal to $50 \%$." By applying VQD-QCD to $\mathrm{DiDi}$, such value expectations are transformed into the configuration scheme for quality/capabilities of fine-grained processes, activities, and resources in DiDi. Examples of GQPs include "Maximum Waiting Time during the Peak Period," "Drivers' Service Attitude," and "Security Guarantee Level," and examples of LQPs/CPs include "Number of Called Taxis," "Radius of Coverage," and "Longest Grab Time for Taxi Drivers."

As mentioned in the previous section, the greatest challenge in the quality/capability design of transboundary services is the complex conflicts/correlations among value expectations, GQPs, LQPs, and CPs. In DiDi, such scenarios exist extensively. For example, the GQP "Maximum Waiting Time during Peak Period" has a quantitative relation with multiple LQPs, "Number of Called Taxis," "Radius of Coverage," and "Longest Grab Time for Taxi Drivers" and the realisation of the GQP depends on a reasonable configuration of these LQPs. In our VQD-QCD, a set of mathematical methods are used for this purpose.

Because of the limited space in this paper, we cannot introduce all the details of VQD-QCD. In the following sections, we use VQD as an example to demonstrate our idea about the quality design of transboundary services. The philosophy of QCD is quite similar to that of VQD, and readers who are interested in it may refer to our other papers. Note that most existing papers on QFD research also focus mainly on the first phase $[5,8]$.

\section{VQD: Design Method for the Optimal GQPs of Transboundary Services}

3.1. Extension of QFD: VQD. As mentioned above, QFD is used to decompose the user demands to match the production requirements layer by layer. We propose an evolved method based on QFD called VQD to translate value expectations of multiple stakeholders into an optimal configuration scheme for GQPs for transboundary services. Before presenting the details of the VQD process, in this section, we explain the approach used to extend QFD to VQD:

(1) Because of the differences between product design and the design of transboundary services, it is necessary to enhance the semantic expressive ability of QFD, for example, how to describe the value expectations and how to describe the constraints on GQPs. By contrast, the analysis methods of QFD must be extended according to the scenario of transboundary service design. For example, it must be able to calculate the competitive priority ratings of VIs based on competitive analysis rather than only the computation of competitive priority ratings of customer demand based on customer competitiveness analysis.

(2) QFD uses 1-to-9 scale to characterise the satisfaction degree of customer demands supported by different product design attributes [6,9]. However, when designing a transboundary service, because of the restrictions of transboundary service capabilities, sometimes it is difficult to obtain a reasonable configuration scheme once; multiple stakeholders need to remake value propositions. Thus, it is necessary to establish quantitative relations between VIs and GQPs, which can greatly ease the transformation from a value expectation of each VI into the optimal configuration scheme for GQPs. In the intervening time, a set of functions, which can characterise the quantitative relations in VQD, is also required.

3.2. VQD-Based Design Process of GQPs. Figure 3 shows the VQD-based design process of GQPs, which is used to generate the configuration scheme for GQPs. Considering the difference and relation between product design and the design of transboundary services, the current nine-step HoQ model [10] in the product design domain is extended to a 10 -step HoQ model. Its steps are described in detail as follows:

Step 1. Identify stakeholders and their value expectations First, the designers must determine the stakeholders that correspond to the VIs of the transboundary service concerned and then gather their value expectations through focus group and individual interviews [6]. The VIs identified are denoted as $\mathrm{VI}=\left\{v i_{1}, v i_{2}, \ldots, v i_{M}\right\}$. The stakeholders make value propositions according to these $M$ VIs, which propose a set of value expectations $\mathrm{VP}=\left\{v p_{1}, v p_{2}, \ldots, v p_{M}\right\}$ on these value $M$ indicators, where each $v p_{i} \in \mathrm{VP}$ is either $v p_{i}=\left(v i_{i}\right.$, $v i_{i-} \mathrm{EV}$, operator $\left.S\right)$ or $v p_{i}=\left(v i_{i},\left\{v i_{i-} \mathrm{EV}_{1}, v i_{i-} \mathrm{EV}_{2}, \ldots\right.\right.$, $\left.v i_{i-} \mathrm{EV}_{k}\right\}$, operator $\left.D\right)$. The former is used to describe continuous VIs, whereas the latter is used for discrete VIs. OperatorS could be, for example, ">," “<," or " $\leq$." For example, when designing DiDi, if value expectation $v p_{1}$ of VI $v i_{1}$ "Market Share" is "larger than $70 \%$ ", then the $v p_{1}$ collected can be denoted as $v p_{1}=\left(v i_{1}, 70 \%,>\right)$. For $v p_{i}=\left(v i_{i},\left\{v i_{i-} \mathrm{EV}_{1}, v i_{i-} \mathrm{EV}_{2}, \ldots, v i_{i-} \mathrm{EV}_{k}\right\}\right.$, operator $D)$, the multiple operands in the set $\left\{v i_{i-} \mathrm{EV}_{1}, v i_{i-} \mathrm{EV}_{2}, \ldots\right.$, $\left.v i_{i-} \mathrm{EV}_{k}\right\}$ are in ascending order, and operator $D$ may be, for example, " $\epsilon_{\text {up }}$ " or " $\epsilon_{\text {down }}$ ". For $v p_{i}=\left(v i_{i}\right.$, $\left.\left\{v i_{i-} \mathrm{EV}_{h}, \ldots, v i_{i-} \mathrm{EV}_{k}\right\}, \epsilon_{\text {up }}\right)$, where $1<h \leq k, " \epsilon_{\text {up }}$ " means that the $v i_{i}{ }^{\prime} s$ value belongs to the set $\left\{v i_{i-} \mathrm{EV}_{h}, \ldots, v i_{i-} \mathrm{EV}_{k}\right\}$ and it should be larger than or equal to $v i_{i-} \mathrm{EV}_{h}$; while for $v p_{i}=\left(v i_{i},\left\{v i_{i-} \mathrm{EV}_{1}, \ldots\right.\right.$, 


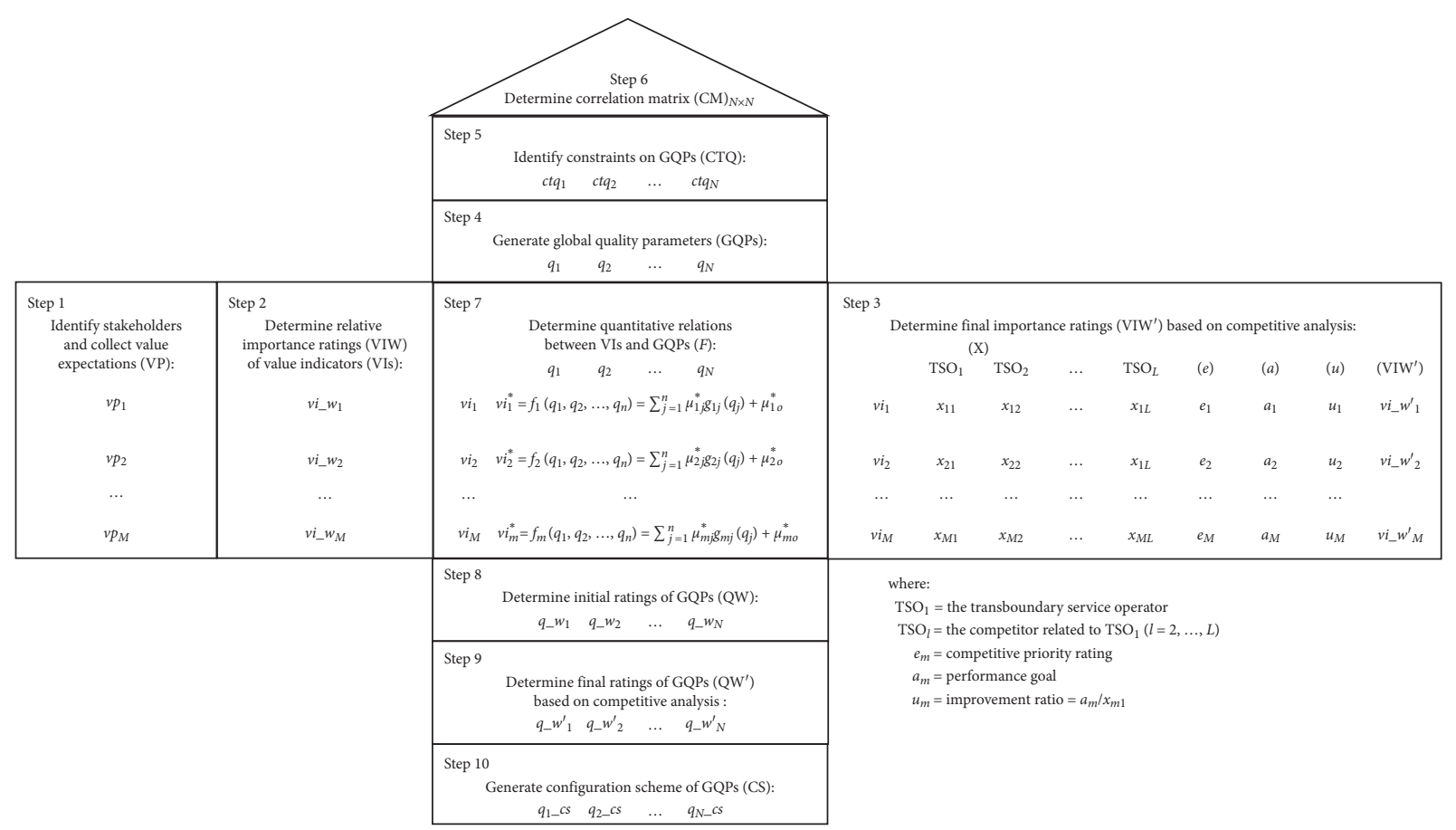

FIGURE 3: VQD-based design process of GQPs for transboundary services.

$\left.\left.v i_{i-} \mathrm{EV}_{j}\right\}, \epsilon_{\text {down }}\right)$, where $1 \leq j<k$, " $\epsilon_{\text {down }}$ " means that the $v i_{i}$ 's value belongs to the set $\left\{v i_{i-} \mathrm{EV}_{1}, \ldots, v i_{i-} \mathrm{EV}_{j}\right\}$ and it should be smaller than or equal to $v i_{i-} \mathrm{EV}_{j}$. For example, if value expectation $v p_{2}$ of VI $v i_{2}$ "Customer Satisfaction" is "larger than or equal to 4 stars," then the $v p_{2}$ collected can be denoted as $v p_{2}=\left(v i_{2},\{4\right.$ stars, 5 stars $\left.\}, \epsilon_{\text {up }}\right)$.

Step 2. Determine the relative importance ratings of VIs The relative importance of the VIs is typically expressed as a set of ratings that can be determined by the stakeholders using their perceptions of the relative importance of these indicators, whereas the stakeholders' perceptions can be obtained by applying a fuzzy analytic hierarchy process (FAHP) [15], which starts from an individual interview or mail survey. Then, the relevant fuzzy complementary judgment matrix and fuzzy consistent judgment matrix are established in turn. Finally, the relative importance ratings of the VIs are computed by normalising the rank aggregation method [16] and are described as $M$ dimensional vector VIW $=\left(v i \_w_{1}, v i \_w_{2}, \ldots, v i \_w_{M}\right)$.

Step 3. Determine the final importance ratings based on competitive analysis

To improve the competitiveness of the transboundary service operator $\left(\mathrm{TSO}_{1}\right)$ after investigating the relevant markets, it is necessary to consider the competitors' impact on vector VIW. Hence, first, it is essential for $\mathrm{TSO}_{1}$ to identify the competitors who provide a similar service. Assume that $L-1$ competitors are identified in the relevant markets, denoted as $\mathrm{TSO}_{2}, \ldots, \mathrm{TSO}_{L}$. Then, it is necessary to collect the stakeholders' perceptions of the relative performance of these $L$ operators' services of a similar type in terms of the $M$ VIs. Thus, the operators' performance ratings on the $M$ VIs can be obtained and denoted as a comparison matrix $X=\left(x_{m l}\right)_{M \times L}$, where $x_{m l}$ is the performance rating of operator $\mathrm{TSO}_{l}$ on $\mathrm{VI} \mathrm{VI}_{m}$.

Based on matrix $X$, the competitive priority ratings on the $M$ VIs for operator $\mathrm{TSO}_{1}$ can be obtained using the entropy method [17] and denoted as $e=\left(e_{1}, e_{2}, \ldots, e_{M}\right)$, where $e_{m}$ represents operator $\mathrm{TSO}_{1}$ 's priority rating on $\mathrm{VI} \mathrm{VI}_{m}$. According to $e$, the performance goals of the $M$ VIs could be set competitively and realistically by operator $\mathrm{TSO}_{1}$ and denoted as $a=\left(a_{1}, a_{2}, \ldots, a_{M}\right)$. Thus, operator $\mathrm{TSO}_{1}$ 's improvement ratio for $\mathrm{VI}_{m}$ is $u_{m}=a_{m} / x_{m 1}$. It is obvious that the more ratio $u_{m}$ improves, the more operator $\mathrm{TSO}_{1}$ should work on VI $\mathrm{VI}_{m}$ and the more important $\mathrm{VI}_{m}$ for operator $\mathrm{TSO}_{1}$. Thus, the final importance ratings of the $M$ VIs for operator $\mathrm{TSO}_{1}$ are determined jointly by the relative importance, competitive priority, and improvement ratio. Additionally, they can be denoted as $\mathrm{VIW}^{\prime}=\left(v i \_w_{1}^{\prime}, v i \_w_{2}^{\prime}, \ldots, v i \_w_{M}^{\prime}\right)$, where $v i \_w_{m}^{\prime}=$ $u_{m} \times v i \_w_{m} \times e_{m}$.

\section{Step 4. Generate GQPs}

The generation of GQPs actually involves solving the problem of which GQPs of transboundary services need to be sufficiently implemented to meet the stakeholders' value expectations. Thus, it should first analyse the existing data of the traditional services in the same domain as the transboundary service and determine which quality parameters in the traditional services may affect the realisation of VIs. Then, through market investigation, it should identify more quality 
parameters that can be used to solve a series of pain points of traditional services in an innovative manner, which aims at achieving the "attack from higher dimensions" and ultimately generating the entire set of GQPs.

During GQP generation, the brainstorming method is first used to realise the primary selection of GQPs based on the above analysis. Second, it needs to identify three relations that may exist between GQPs, that is, inclusion relationship, cross relationship, and independent relationship, and remove redundant GQPs. Furthermore, from the perspective of correlation, it is necessary to qualitatively identify another four relations that may exist, that is, positive correlation, negative correlation, mutual exclusion, and uncorrelation, and eliminate mutual exclusion. Thus far, a set of GQPs that affects the implementation of the VI has been generated and denoted as GQP $=\left(q_{1}, q_{2}, \ldots, q_{N}\right)$.

\section{Step 5. Identify constraints on GQPs}

When identifying the constraints on GQPs, the designers have to consider the value constraints of GQPs themselves, which can be identified by analysing the existing data (i.e., the available quality of activities and capability of resources that the stakeholders can offer in the real world). For the continuous quality parameter, there are $q_{j} \in \mathrm{GQP}$ and $c q_{j}=\left[q_{j} \mathrm{LP}, q_{j} \mathrm{UP}\right]$, where $q_{j}$ LP represents the lower bound of the constraint on the $q_{j}$ and $q_{j-}$ UP represents the upper bound of the $q_{j}$ s constraint; for discrete quality parameters, there are $q_{j} \in \mathrm{GQP}$ and $c q_{j}=\left\{q_{j} \mathrm{LP}, \ldots, q_{j-} V_{j}, \ldots, q_{j-} \mathrm{UP}\right\}$.

By contrast, the designers also have to consider the constraints of the relationships between GQPs. In Step 5, the designers only need to consider the qualitative relational constraints caused by domain knowledge, policies, and business regulations, whereas the quantitative constraints of the relationships between GQPs are identified in Step 6. For qualitative constraints, the designers need to identify the necessary relationships between GQPs. For example, when improving $\mathrm{DiDi}$, the quality parameter "Security Guarantee Level" is a necessary condition that relates to the other quality parameters.

Step 6. Determine the correlation matrix among GQPs The optimal configuration scheme for GQPs is affected not only by the constraints obtained in Step 5 but also by the value of the relevant GQPs. There is a correlation among GQPs, which can be expressed as the correlation matrix. Through the FLAR method, we use the collected data combined with expert knowledge to obtain the correlation matrix among GQPs. More details are provided in Section 4.

Step 7. Determine the quantitative relations between VIs and GQPs

For each $\mathrm{VI} \mathrm{VI}_{m}$, the designers need to determine the value range of the quality parameters related to $\mathrm{VI}_{m}$ and the $\mathrm{VI}_{m}$ 's own value range according to the actual scenario. They also need to determine the degree of VI $\mathrm{VI}_{m}$ that would be implemented according to different values of quality parameters. Therefore, through the FLAR method, we determine the quantitative relations between VIs and GQPs by collecting data and expert knowledge. The same method is used in Steps 6 and 7, and more details are provided in Section 4.

Step 8. Determine the initial ratings of GQPs

The initial ratings of GQPs are determined by two factors, the final importance ratings of VIs and the quantitative relations between VIs and GQPs, so the input of Step 8 is the output of Steps 3 and 7. In Step 7, we obtain the quantitative relations between VIs and GQPs. In the expressions of the quantitative relations, there exist the influence coefficient vectors of quality parameters on VIs, and the influence coefficient vectors reflect the strength of correlations between quality parameters and VIs. The greater the influence coefficient, the greater the influence of the corresponding quality parameter change on the VI.

The initial ratings of GQPs indicate the basic importance of the GQPs designed in relation to the VIs. After the normalisation of the influence coefficient vector, the initial ratings of GQPs can be obtained by combining the output of Step 3 and the normalised results. They can be computed as the simple weighted average over their relationships with the VIs: $q \_w_{n}=v i \_w_{1}^{\prime} \times v i_{1 n}^{*}+v i \_w_{2}^{\prime} \times v i_{2 n}^{*}+\cdots+v i \_w_{M}^{\prime} \times$ $v i_{M n}^{*}, n=1,2, \ldots, N$.

Step 9. Determine the final ratings of GQPs based on competitive analysis

Step 9 can also be performed by investigating the relevant markets. Although some quality parameters of the competitors' service cannot be easily obtained, careful quality assessments should still be conducted to provide reliable ratings that represent the quality performance of the competitors' service in terms of the $N$ GQPs. Thus, the operators' performance ratings for the $N$ GQPs can be obtained and denoted as a comparison matrix $Y=\left(y_{m l}\right)_{N \times L}$, where $y_{n l}$ is the quality performance rating of operator $\mathrm{TSO}_{l}$ on GQP $q_{n}$.

Based on matrix $Y$, the competitive priority ratings on the $N$ GQPs for operator $\mathrm{TSO}_{1}$ can be obtained using the entropy method [17] and denoted by $z=\left(z_{1}, z_{2}, \ldots\right.$, $z_{N}$ ), where $z_{n}$ represents operator $\mathrm{TSO}_{1}$ 's quality competitive priority with respect to the competitors' quality performance ratings. According to $z$, the performance goals of the $N$ GQPs could also be set by operator $\mathrm{TSO}_{1}$ and denoted by $b=\left(b_{1}, b_{2}, \ldots, b_{N}\right)$. Thus, operator $\mathrm{TSO}_{1}$ 's improvement ratios for $q_{n}$ is $v_{n}=b_{n} / y_{n 1}\left(b_{n}\right.$ is a positive quality parameter) and $y_{n 1} /$ $b_{n}$ ( $b_{n}$ is a negative quality parameter). Thus, the final ratings of the $N$ GQPs for operator $\mathrm{TSO}_{1}$ are determined jointly by the initial ratings, competitive priority, and improvement ratio. Additionally, they can be denoted by $\mathrm{QW}^{\prime}=\left(q_{-} w_{1}^{\prime}, q_{-} w_{2}^{\prime}, \ldots, q_{-} w_{N}^{\prime}\right)$, where q_ $w_{n}^{\prime}=v_{n} \times$ q_ $w_{n} \times z_{n}$.

Step 10. Generate the configuration scheme for GQPs The input of step 10 is the output of Steps 1, 3, 5, 6, and 7. Based on these inputs, a method for generating a 
configuration scheme for GQPs is proposed. In this method, the constrained target optimisation model (i.e., fuzzy nonlinear programming model) is established to solve the problem of generating the configuration scheme. Then, the concept of confidence is used to transform the fuzzy linear programming model into two linear programming models. Next, the two linear programming models are solved easily. Finally, the optimal configuration scheme for GQPs is obtained. The details of Step 10 are provided in Section 5.

\section{Method for Determining the Correlation Matrix and the Quantitative Relations between VIs and GQPs}

\subsection{Influence Factor Analysis and Determining the Influence} Degree Relation Function. To meet value expectations VP= $\left\{v p_{1}, v p_{2}, \ldots, v p_{m}\right\}$ stated by the stakeholders of transboundary services for VIs $\mathrm{VI}=\left\{v i_{1}, v i_{2}, \ldots, v i_{m}\right\}$, it is necessary to generate an appropriate configuration scheme for GQPs for transboundary services. Therefore, it is necessary to first identify the relationships between VIs and GQPs. Specifically, this involves determining the quantitative relations between both VIs and GQPs, which can be expressed as a set of weight relation functions $F=\left\{f_{1}, f_{2}, \ldots, f_{m}\right\}$, where $v i_{i}=f_{i}\left(q_{1}, q_{2}, \ldots, q_{n}\right)$.

When determining function $f_{i}$ specifically, it is necessary to analyse which characteristics of GQPs are related to the realisation of VIs: (1) types of GQPs: different types of GQPs have different ranges and units of measurement, and their influence on the realisation of VIs varies greatly; (2) values of GQPs: the change of the same GQP in different value ranges has a different influence on the realisation of VIs; and (3) correlation among GQPs: different correlations among GQPs have different influences on the realisation of VIs.

Through the above analysis, we find that if we want to determine function $f_{i}$, we should first describe the influence of the value of a single quality parameter on the realisation of a single VI when other relevant quality parameters remain unchanged. Specifically, this involves determining the quantitative relations between a single $\mathrm{VI} v i_{i}$ and each quality parameter $q_{j}$, which can be expressed as a set of one-to-one influence degree relation functions $G=\left\{g_{i 1}, g_{i 2}, \ldots, g_{i n}\right\}$, where $v i_{i-} d=g_{i j}\left(q_{j}\right)$ and variable $v i_{i-} d$ is the influence degree of the quality parameter $q_{j}$ 's change on VI $v i_{i}$. The purpose of function $g_{i j}$ is to convert the values of quality parameters of different dimensions into the unified values of influence degree $v i_{i-} d$, which facilitates the determination of weight relation function $f_{i}$ in the next step. A set of typical influence degree relation functions are given as follows:

Table 2 presents the basic types of one-to-one quantitative relation between quality parameter $q_{j}$ and influence degree $v i_{i-} d$ from multiple angles (e.g., linear and nonlinear angles and positive and negative angles). Additionally, the more complex quantitative relation between both $q_{j}$ and $v i_{i-} d$ can be obtained by combining the basic types of relationships. For example, when quality parameter $q_{j}$ "Quantity of Available Taxis" of DiDi is significantly lower than the average level of competitors in the relevant market, the relationship between $q_{j}$ and influence degree $v i_{i} d$ related to the VI "Market Share" is consistent with Type 1. When the value of $q_{j}$ is close to or even far exceeds the average level of competitors, the relationship between $q_{j}$ and $v i_{i-} d$ is consistent with Type 2 . Therefore, the relationship between both of them is an S-type quantitative relation as a whole.

Type 1: It is an increasing function whose derivative increases gradually. The degree of influence on the VI increases with the improvement of the quality parameter, and the rate of increase accelerates.

Type 2: It is an increasing function whose derivative decreases gradually. The degree of influence on the VI increases with the improvement of the quality parameter, and the rate of increase decelerates.

Type 3: It is a decreasing function whose derivative increases gradually. The degree of influence on the VI decreases with the improvement of the quality parameter, and the rate of decrease decelerates.

Type 4: It is a decreasing function whose derivative decreases gradually. The degree of influence on the VI decreases with the improvement of the quality parameter, and the rate of decrease accelerates.

For the above four types, $b>0$, and the greater the value of $b$, the greater the curvature of the function.

Type 5: It is an increasing function whose derivative is constant. The degree of influence on the VI increases with the improvement of the quality parameter, and the rate of increase is constant, where $k>0$.

Type 6: It is a decreasing function whose derivative is constant. The degree of influence on the VI decreases with the improvement of the quality parameter, and the rate of reduction is constant, where $k>0$.

Next, because different correlations among GQPs have different influence degrees on the realisation of VIs, to generate an appropriate configuration scheme for GQPs, a set of correlation functions needs to be described as $H=\left\{h_{1}\right.$, $\left.h_{2}, \ldots, h_{n}\right\}$, where $q_{j}=h_{j}\left(q_{1}, q_{2}, \ldots, q_{j-1}, q_{j+1}, \ldots, q_{n}\right)$. This is similar to the idea of determining weight relationship function $f_{i}$ when determining the correlation function $h_{j}$. It is also necessary to first determine the one-to-one influence degree relation function of a pair of GQPs using correlation. It should be noted that the type of influence degree relation function related to function $h_{j}$ is the same as the type of function in Table 2, but the meanings of the independent variable and dependent variables of the function are different.

Specifically, the influence degree relation function related to function $h_{j}$ can be expressed as $G=\left\{g_{j 1}, g_{j 2}, \ldots\right.$, $\left.g_{j-1}, g_{j+1}, \ldots, g_{j n}\right\}$, where $q_{j} d=g_{j k}\left(q_{k}\right)$, which means that $q_{j}$ has a correlation with $q_{k}, q_{k}$ is the independent variable of function $g_{j k}$, and $q_{j \_} d$ is the dependent variable, and the influence degree of $q_{k}$ 's change on quality parameter $q_{j}$. The purpose of function $g_{j k}$ is the same as that of the above function $g_{i j}$.

Given the above, the realisation of the VIs is the result of the comprehensive influence of all the changes of the relevant quality parameters. Now, function $g_{i j}$ and function $g_{j k}$ have been determined, that is, the influence degree of quality 
TABLe 2: Typical influence degree relation functions.

\begin{tabular}{lcl}
\hline Type of function & Formula of function & Image of function \\
Type 1 & $v i_{i-} d=\left(1 /\left(e^{b}-1\right)\right) \cdot e^{b \cdot\left(\left(q_{j}-q_{j}^{l p}\right) /\left(q_{j}^{u p}-q_{j}^{l p}\right)\right)}-\left(1 /\left(e^{b}-1\right)\right)$
\end{tabular}

Type 2

$$
v i_{i-} d=-\left(e^{b} /\left(e^{b}-1\right)\right) \cdot e^{-b \cdot\left(\left(q_{j}-q_{j}^{l p}\right) /\left(q_{j}^{u p}-q_{j}^{l p}\right)\right)}+\left(e^{b} /\left(e^{b}-1\right)\right)
$$
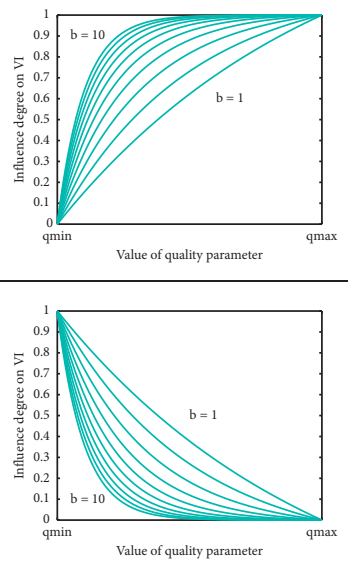

Type 3

$$
v i_{i-} d=\left(e^{b} /\left(e^{b}-1\right)\right) \cdot e^{-b \cdot\left(\left(q_{j}-q_{j}^{l p}\right) /\left(q_{j}^{u p}-q_{j}^{l p}\right)\right)}-\left(1 /\left(e^{b}-1\right)\right)
$$

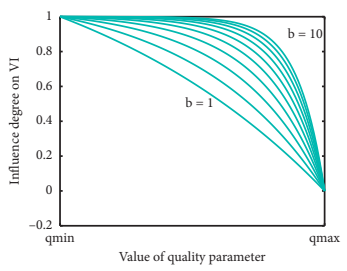

Type 4

$$
v i_{i-} d=-\left(1 /\left(e^{b}-1\right)\right) \cdot e^{b \cdot\left(\left(q_{j}-q_{j}^{l p}\right) /\left(q_{j}^{u p}-q_{j}^{l p}\right)\right)}+\left(e^{b} /\left(e^{b}-1\right)\right)
$$

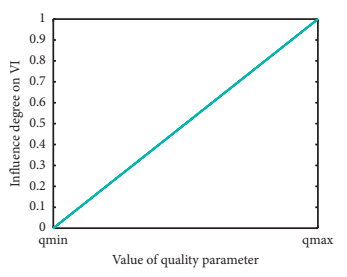

Type 5

$$
v i_{i-} d=k q_{j}+b
$$

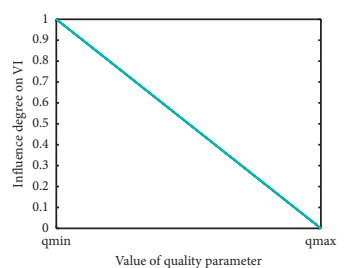

Type 6

$$
v i_{i-} d=-k q_{j}+b
$$

parameter $q_{j}$ 's change on VI $v i_{i}$ can be identified and the influence degree of $q_{k}$ 's change on quality parameter $q_{j}$ can also be identified. Then, the weight relation function and correlation function can be constructed.

\subsection{Method for Constructing the Weight Relation Function} and Correlation Function. Machine learning paradigms (e.g., decision tree, hidden Markov model, and regression analysis) perform extremely well in identifying the relationships between multiple variables [18]. Considering the inherent vagueness and uncertainty of relationships between VIs related to value expectations and GQPs, in addition to those among GQPs $[10,19]$, we adopt the fuzzy regression method to determine weight relation function $f_{i}$ and correlation function $h_{j}$.

Choi and Buckley [20] indicated that, in general regression models, the least absolute deviation estimation is more effective than the least squares method. In regression analysis, least squares estimation is mostly used. Least squares estimation performs poorly when the data contain 
some outliers, whereas least absolute deviation estimation is more effective than least squares estimation in linear and nonlinear regression. Therefore, for the estimation of the fuzzy regression coefficient, we believe that the minimum absolute deviation estimation using one norm is better than least squares estimation using two norms; Zeng's experiment [13] on FLAR also proved this conclusion. Therefore, we use FLAR to determine weight relation function $f_{i}$ and correlation function $h_{j}$.

4.2.1. FLAR and Sample Set Collection. The FLAR model can define the absolute distance between fuzzy numbers so that the fuzzy regression problem can be transformed into the problem of minimising the fuzzy distance. Using FLAR, the problem in this section can be transformed into the problem of minimising the error between the observed value of the corresponding variable and its estimated value, thereby making the quantitative relation obtained as close as possible to the actual scenario.

The input of FLAR is $l$ sets of reliable and practical data: $\left(X_{1}, y_{1}^{*}\right),\left(X_{1}, y_{2}^{*}\right), \ldots,\left(X_{r}, y_{r}^{*}\right), \ldots,\left(X_{l}, y_{l}^{*}\right)$, where $X_{r}=$ $\left(x_{1 r}, x_{2 r}, x_{3 r}, \ldots, x_{n r}\right)$ and $y_{r}^{*}=\left(y_{r}^{c}, y_{r}^{s}\right), r=1,2,3, \ldots, l$. Additionally, its output is the fuzzy regression coefficient $\mu^{*}=$ $\left(\mu_{0}^{*}, \mu_{1}^{*}, \mu_{2}^{*}, \ldots, \mu_{n}^{*}\right)$ of the regression equation of $y$ with respect to $X$. In FLAR, the distance between symmetrical triangular fuzzy members $A=\left(c_{1}, s_{1}\right)$ and $B=\left(c_{2}, s_{2}\right)$ can be expressed as $d=\left|c_{1}-c_{2}\right|+\left|s_{1}-s_{2}\right|$. The problem is converted to minimising the sum of errors between $y_{r}^{*}$ and the estimated value of the corresponding variable and can be denoted as

$$
\begin{aligned}
d_{\min }= & \sum_{r=1}^{l}\left(\left|y_{r}^{c}-x_{1 r} \mu_{1}^{c}-x_{2 r} \mu_{2}^{c}-\cdots-x_{n r} \mu_{n}^{c}-\mu_{0}^{c}\right|\right. \\
& \left.+\left|y_{r}^{s}-x_{1 r} \mu_{1}^{s}-x_{2 r} \mu_{2}^{s}-\cdots-x_{n r} \mu_{n}^{s}-\mu_{0}^{s}\right|\right) .
\end{aligned}
$$

For equation (1), we can make the following transformation:

$$
\begin{aligned}
\left|y_{r}^{c}-x_{1 r} \mu_{1}^{c}-x_{2 r} \mu_{2}^{c}-\cdots-x_{n r} \mu_{n}^{c}-\mu_{0}^{c}\right| & =\alpha_{r}^{c}+\beta_{r}^{c} \\
y_{r}^{c}-x_{1 r} \mu_{1}^{c}-x_{2 r} \mu_{2}^{c}-\cdots-x_{n r} \mu_{n}^{c}-\mu_{0}^{c} & =\alpha_{r}^{c}-\beta_{r}^{c} \\
\left|y_{r}^{s}-x_{1 r} \mu_{1}^{s}-x_{2 r} \mu_{2}^{s}-\cdots-x_{n r} \mu_{n}^{s}-\mu_{0}^{s}\right| & =\alpha_{r}^{s}+\beta_{r}^{s} \\
y_{r}^{s}-x_{1 r} \mu_{1}^{s}-x_{2 r} \mu_{2}^{s}-\cdots-x_{n r} \mu_{n}^{s}-\mu_{0}^{s} & =\alpha_{r}^{s}-\beta_{r}^{s} \\
\alpha_{r}^{c} \geq 0, \beta_{r}^{c} \geq 0, \alpha_{r}^{s} \geq 0, \beta_{r}^{s} \geq 0, r & =1,2,3, \ldots, l,
\end{aligned}
$$

where $\alpha_{r}^{c}, \beta_{r}^{c}, \alpha_{r}^{S}$, and $B_{r}^{s}$ are nonnegative real numbers.

Thus, the original problem is transformed into solving the linear programming model:

$$
\begin{aligned}
d_{\min } & =\sum_{r=1}^{l}\left(\alpha_{r}^{c}+\beta_{r}^{c}+\alpha_{r}^{s}+\beta_{r}^{s}\right), \\
& \left\{\begin{array}{l}
y_{r}^{c}-x_{1 r} \mu_{1}^{c}-x_{2 r} \mu_{2}^{c}-\cdots-x_{n r} \mu_{n}^{c}-\mu_{0}^{c}=\alpha_{r}^{c}+\beta_{r}^{c}, \\
y_{r}^{s}-x_{1 r} \mu_{1}^{s}-x_{2 r} \mu_{2}^{s}-\cdots-x_{n r} \mu_{n}^{s}-\mu_{0}^{s}=\alpha_{r}^{s}+\beta_{r}^{s}, \\
\alpha_{r}^{c} \geq 0, \beta_{r}^{c} \geq 0, \alpha_{r}^{s} \geq 0, \beta_{r}^{s} \geq 0, r=1,2,3, \ldots, l, \\
\mu_{i}^{c} \geq 0, \mu_{i}^{s} \geq 0, i=1,2,3, \ldots, n .
\end{array}\right.
\end{aligned}
$$

When collecting the sample set, it is first necessary to derive $L$ sets of values of $N$ GQPs in line with actual conditions through communication with transboundary service designers and domain experts. The designers and domain experts should have a very good understanding of transboundary services and can provide $L$ sample sets according to their accumulated rich personal experience and domain knowledge. The values of $N$ GQPs are denoted as $Q_{r}=\left(q_{1 r}, q_{2 r}, q_{3 r}, \ldots, q_{i r}, \ldots, q_{n r}\right)$, where $r=1,2, \ldots, L$ and $q_{i r}$ represents the observed value of the $i$ th GQP in the $r$ th sample set and is accurate data.

Then, it is necessary to derive the observed values of $M$ VIs for each $Q_{r}$, which is denoted as $p v i_{i r}^{*}=\left(p v i_{i r}^{c}, p v i_{i r}^{s}\right)$, where $r=1,2, \ldots, L$ and $i=1,2, \ldots, M$. Because the observed values of $M$ VIs are determined by designers based on the observed values of the relevant GQPs, they are subjective and imprecise. Thus, fuzzy numbers should be assigned to the $M$ VIs' observed values so that their vagueness can be captured, where $p v i_{i r}^{*}$ represents the observed value of the $i$ th VI with respect to $Q_{r}$ and is a fuzzy number. Centre point value $p v i_{i r}^{c}$ describes the most likely value of $p v i_{i r}^{*}$, whereas the spread $p v i_{i r}^{s}$ describes the precision of $p v i_{i r}^{*}$. Thus, we collect the $l$ sets of sample data that contain the quantitative relations between GQPs and VIs and conform to the correlations among GQPs.

4.2.2. FLAR-Based Method for Constructing Functions $f_{i}$ and $h_{j}$. The FLAR model is applied to construct weight relation function $f_{i}$, and the formulas of function $f_{i}$ are given as follows:

$$
v i_{i}^{*}=f_{i}\left(q_{1}, q_{2}, \ldots, q_{n}\right)=\sum_{j=1}^{n} \mu_{i j}^{*} g_{i j}\left(q_{j}\right)+\mu_{i o}^{*} .
$$

In equation (4), $q_{1}, q_{2}, \ldots, q_{n}$ represent the input vector, where $q_{j}$ represents the crisp input value of the $j$ th GQPs; $q_{1}, q_{2}$, $q_{3}, \ldots, q_{j_{0}}$ represent continuous GQPs and $q_{j_{0}+1}, q_{j_{0}+2}, \ldots, q_{n}$ represent the discrete GQPs; $v i_{i}^{*}$ represents the fuzzy output value of the $i$ th VI; and $\mu_{i}^{*}=\left(\mu_{i 0}^{*}, \mu_{i 1}^{*}, \mu_{i 2}^{*}, \ldots, \mu_{i n}^{*}\right)$ is the fuzzy coefficient vector that needs to be determined when constructing function $f_{i}$. In this paper, the construction problem of function $f i$ is transformed into the following: input $l$ sets of data $\left\{\left(Q_{1}, p v i_{i 1}^{*}\right),\left(Q_{2}, p v i_{i 2}^{*}\right), \ldots,\left(Q_{l}, p v i_{i l}^{*}\right)\right\}$ and then determine the fuzzy coefficient vector $\left(\mu_{i 0}^{*}, \mu_{i 1}^{*}, \mu_{i 2}^{*}, \ldots, \mu_{i n}^{*}\right)$ so that output value $v i_{i}^{*}$ of VI calculated by equation (4) has the best fitting influence on declared value $p v i_{i}^{*}$ of the VI.

Let $Q_{r}=\left(q_{1 r}, q_{2 r}, \ldots, q_{n r}\right)$ denote the accurate input vector of the $r$ th group and $v i_{i r}^{*}$ represent the fuzzy output of VI calculated by equation (4) after $Q_{r}$ is input. $v i_{i r}^{*}$ is a symmetric triangular fuzzy number, which can be expressed as $v i_{i r}^{*}=\left(v i_{i r}^{c}, v i_{i r}^{s}\right)$, where $v i_{i r}^{c}$ represents the centre point value of fuzzy number $v i_{i r}^{*}$, which means the most likely value, and $v i_{i r}^{s}$ represents the spread of fuzzy number $v i_{i r}^{*}$, which means the precision of $v i_{i r}^{*}$. Let $v i_{i r}^{\max }=v i_{i r}^{c}+v i_{i r}^{s}$ and $v i_{i r}^{\min }=v i_{i r}^{c}-v i_{i r}^{s}$ represent the upper and lower limits of the fuzzy number, respectively, and $p v i_{i r}^{*}$ represent the declared value of VIs that correspond to the input vector of the $r$ th group. $p v i_{i r}^{*}$ and fuzzy coefficient $\mu_{i j}^{*}$ are also symmetric triangular fuzzy numbers, which have the same meaning as $v i_{i r}^{*}$ and can be expressed as $\left(p v i_{i r}^{*}=p v i_{i r}^{c}, p v i_{i r}^{s}\right)$ and $\mu_{i j}^{*}=$ $\left(\mu_{i j}^{c}, \mu_{i j}^{s}\right)$, respectively. 
The objective function and constraints for solving the problem of constructing weight relationship function $f_{i}$ are given as follows:

$$
\begin{aligned}
\min d= & \sum_{r=1}^{l}\left|p v i_{i r}^{*}-v i_{i r}^{*}\right|=\sum_{r=1}^{l}\left(\left|p v i_{i r}^{c}-\left(\sum_{j=1}^{n} \mu_{i j}^{c} g_{i j}\left(q_{j r}\right)+\mu_{i o}^{c}\right)\right|\right. \\
& \left.+\left|p v i_{i r}^{s}-\left(\sum_{j=1}^{n} \mu_{i j}^{s} g_{i j}\left(q_{j r}\right)+\mu_{i o}^{s}\right)\right|\right),
\end{aligned}
$$$$
\text { s.t. } \quad \mu_{i j}^{s} \geq 0, \mu_{i j}^{c} \geq 0, \quad j=1,2, \ldots, n \text {. }
$$

To facilitate the calculation, the mathematical model is transformed into the following linear programming model:

$$
\begin{aligned}
& \min d=\sum_{r=1}^{l}\left(\mu_{r}+v_{r}+\omega_{r}+\delta_{r}\right), \\
& \text { s.t. } \quad\left\{\begin{array}{l}
v i_{i r}^{c}-\left(\sum_{j=1}^{n} \mu_{i j}^{c} g_{i j}\left(q_{j r}\right)+\mu_{i o}^{c}\right)=\mu_{r}-v_{r}, \\
v i_{i r}^{s}-\left(\sum_{j=1}^{n} \mu_{i j}^{s} g_{i j}\left(q_{j r}\right)+\mu_{i o}^{s}\right)=\omega_{r}-\delta_{r}, \\
\mu_{r} \geq 0, v_{r} \geq 0, \omega_{r} \geq 0, \delta_{r} \geq 0, r=1,2,3, \ldots, l, \\
\mu_{i j}^{s} \geq 0, \mu_{i j}^{c} \geq 0, j=1,2,3, \ldots, n .
\end{array}\right.
\end{aligned}
$$

The above linear programming model can be solved using a MATLAB program, and then the fuzzy coefficient set $\mu_{i}^{*}=\left(\mu_{i 0}^{*}, \mu_{i 1}^{*}, \mu_{i 2}^{*}, \ldots, \mu_{i n}^{*}\right)$ can be obtained, that is, the construction of the weight relation function $f_{i}$ is complete.

The construction method of correlation function $h_{j}$ is similar to that explained above, and its expression is as follows:

$$
q_{j}^{*}=h_{j}\left(q_{1}, q_{2}, \ldots, q_{j-1}, q_{j+1}, \ldots, q_{n}\right)=\sum_{k=1 \& k \neq j}^{n} \alpha_{j k}^{*} g_{j k}\left(q_{j}\right)+\alpha_{j o}^{*},
$$

where $\left(q_{1}, q_{2}, \ldots, q_{j-1}, q_{j+1}, \ldots, q_{n}\right)$ represents the input vector, $q_{j}^{*}$ represents the fuzzy output of the $j$ th quality parameter, and $\alpha_{j}^{*}=\left(\alpha_{j 0}^{*}, \alpha_{j 1}^{*}, \alpha_{j 2}^{*}, \ldots, \alpha_{j, j-1}^{*}, \alpha_{j, j+1}^{*}, \ldots, \alpha_{j n}^{*}\right)$ is the fuzzy coefficient vector that needs to be determined when constructing the function $h_{j}$. Fuzzy coefficient $\alpha_{j k}^{*}$ is a symmetric triangular fuzzy number, which can be expressed as $\alpha_{j k}^{*}=\left(\alpha_{j k}^{c}, \alpha_{j k}^{s}\right)$. Then, the mathematical model for solving the construction of correlation function $h_{j}$ is as follows:

$$
\begin{aligned}
\min d= & \sum_{r=1}^{l}\left|q_{j r}-q_{j r}^{*}\right|=\sum_{r=1}^{l}\left(\left|q_{j r}-\sum_{k=1 \& k \neq j}^{n} \alpha_{j k}^{c} g_{j k}\left(q_{k r}\right)+\alpha_{j o}^{c}\right|\right. \\
& \left.+\left|\sum_{k=1 \& k \neq j}^{n} \alpha_{j k}^{s} g_{j k}\left(q_{k r}\right)+\alpha_{j o}^{s}\right|\right),
\end{aligned}
$$$$
\text { s.t. } \quad \alpha_{j k}^{s} \geq 0, \alpha_{j k}^{c} \geq 0 \text {, }
$$

where $q_{j r}$ represents the actual crisp value of the $j$ th quality parameter when inputting the $r$ th vector and $q_{j r}^{*}$ represents the fuzzy output value that corresponds to the input vector of the $r$ th group. Similar to the process of solving function $f_{i}$, the mathematical model is first converted into a linear programming model, and then a MATLAB program is used to solve it. Thus, fuzzy coefficient set $\alpha_{j}^{*}=\left(\alpha_{j 0}^{*}\right.$, $\left.\alpha_{j 1}^{*}, \alpha_{j 2}^{*}, \ldots, \alpha_{j, j-1}^{*}, \alpha_{j, j+1}^{*}, \ldots, \alpha_{j n}^{*}\right)$ can be obtained, that is, the construction of correlation function $h_{j}$ is complete.

\section{Generation of the Configuration Scheme for GQPs}

5.1. Constraints for Generating the Configuration Scheme. When generating the configuration scheme for GQPs according to the quantitative relations between the VIs and the GQPs obtained in Section 4, three aspects of constraints should be considered: (1) the value expectations should be met, (2) the correlation among GQPs should be considered, and (3) the value constraints of GQPs should be satisfied. Therefore, the constraints of the mathematical model for generating the configuration scheme are given as follows:

(1) Cons 1. Constraints related to the value expectations:

$$
\left\{\begin{array}{l}
\sum_{j=1}^{n} \mu_{i j}^{*} g_{i j}\left(q_{j}\right)+\mu_{i_{0}}^{*} \geq v i_{i-} \mathrm{EV}, \quad i=1,2,3, \ldots, i_{0}, \\
\sum_{j=1}^{n} \mu_{i j}^{*} g_{i j}\left(q_{j}\right)+\mu_{i_{0}}^{*} \leq v i_{i-} \mathrm{EV}, \quad i=i_{0}+1, i_{0}+2, i_{0}+3, \ldots, m,
\end{array}\right.
$$

where it is assumed that $0 \leq i_{0} \leq m$; when $i \leq i_{0}$, operator $S$ of $v p_{i}$ is " $>$ " or " $\geq$ " and when $i \geq i_{0}$, operator $S$ of $v p_{i}$ is "<" or " $\leq$."

(2) Cons 2. Constraints related to the correlation among GQPs:

$$
q_{j}=\sum_{k=1 \& k \neq j}^{n} \alpha_{j k}^{*} g_{j k}\left(q_{j}\right)+\alpha_{j 0}^{*}, \quad j=1,2, \ldots, n .
$$

(3) Cons 3. Constraints related to the value ranges of GQPs:

$$
\left\{\begin{array}{l}
q_{j} \in\left[t q_{j-} \mathrm{LP}, t q_{j-} \mathrm{UP}\right], \quad j=1,2,3, \ldots, j_{0}, \\
q_{j} \in\left\{t q_{j-} \mathrm{LP}, \ldots, t q_{j-} V_{j}, \ldots, t q_{j-} \mathrm{UP}\right\} \\
j=j_{0}+1, j_{0}+2, \ldots, n .
\end{array}\right.
$$

The above constraints determine the feasible region of GQPs. It can be seen that there are fuzzy numbers in equations (9) and (10). When solving the mathematical model to generate the configuration scheme for GQPs, to improve the solution efficiency, it is necessary to convert these fuzzy numbers into crisp numbers.

First, it is necessary to introduce confidence $\lambda$ to convert fuzzy numbers into interval numbers in equation (9). For fuzzy number $A=\left(a_{c}, a_{s}\right)$, the confidence interval with confidence $\lambda$ is expressed as the following formula:

$$
(\underline{A}, \bar{A})=\left(a_{c}-a_{s}+\lambda a_{s}, a_{c}+a_{s}-\lambda a_{s}\right),
$$


where $A$ is called the lower bound of the confidence interval and $\bar{A}$ is called the upper bound of the confidence interval.

For Cons 1, when the operator $S$ is " $>$ " or " $\geq$," $\mu_{i j}^{*}=\mu_{i j}^{*}$; and when operator $S$ is " $<$ " or " $\leq$, , $\mu_{i j}^{*}=\overline{\mu_{i j}^{*}}$. Thus, equation (9) is converted into the following form:

$$
\begin{cases}\sum_{j=1}^{n} \underline{\mu_{i j}^{*}} g_{i j}\left(q_{j}\right)+\underline{\mu_{i_{0}}^{*}} \geq v i_{i-} \mathrm{EV}, & i=1,2,3, \ldots, i_{0}, \\ \sum_{j=1}^{n} \overline{\mu_{i j}^{*}} g_{i j}\left(q_{j}\right)+\overline{\mu_{i_{0}}^{*}} \leq v i_{i-} \mathrm{EV}, & i=i_{0}+1, i_{0}+2, i_{0}+3, \ldots, m, \\ \underline{\underline{\mu_{i j}^{*}}=\mu_{i j}^{c}-\mu_{i j}^{s}+\lambda \mu_{i j}^{s},} & j=0,1,2,3, \ldots, n, \\ \overline{\mu_{i j}^{*}}=\mu_{i j}^{c}+\mu_{i j}^{s}-\lambda \mu_{i j}^{s}, & j=0,1,2,3, \ldots, n .\end{cases}
$$

Provided the configuration scheme for GQPs can satisfy the constraints of equation (13), it can satisfy those of equation (9) because equation (13) is a special case of equation (9).

For Cons 2, because it represents the equality constraints with fuzzy numbers, taking the time complexity and solution accuracy of the method for generating the configuration scheme for GQPs into account comprehensively, it could just retain centre point value $\alpha_{j k}^{c}$ of fuzzy number $\alpha_{j k}^{*}$. This is because when the fuzzy nonlinear programming problem contains $n$ (more than one) equality constraints with fuzzy numbers, a large number of programming models need to be considered, but the final improvement in accuracy is limited. Considering the practical application scenarios in this paper, it is more important to improve the speed of solving the mathematical model on the premise that the solution can achieve a particular accuracy. Thus, equation (10) is converted into the following form:

$$
q_{j}=\sum_{k=1 \& k \neq j}^{n} \alpha_{j k}^{c} g_{j k}\left(q_{j}\right)+\alpha_{j 0}^{c}, \quad j=1,2, \ldots, n .
$$

Equation (14) is a special case of equation (10). Equations (11), (13), and (14) determine the final feasible region of GQPs, in which, regardless of what the fuzzy numbers are in the fuzzy intervals, the configuration scheme for GQPs can meet the value expectations.

\subsection{Method for Generating the Configuration Scheme.} Quality and capability design for transboundary services is an optimisation problem. Similar to the traditional service composition optimisation problem [21], the design of the objective function [22] has a great influence on the result of the optimisation problem. Because the final feasible region is too large to provide guiding suggestions with reference value for designers, it is necessary to determine the detailed configuration scheme for GQPs. "Input Cost" is one key VI when designing transboundary services, and it is also the VI that most concerns all participants. Thus, a method is provided to generate the optimisation configuration scheme for GQPs under the conditions of maximum cost and minimum cost in the feasible region. This can represent two extreme scenarios: one is inputting the highest cost to pursue the best fulfilment of the value expectations of the participants for transboundary services, and the other is pursuing the minimum input cost on the premise of the worst fulfilment of the value expectations. The above two scenarios are solved separately as follows.

"Input Cost" is denoted as $v i_{k}$ and its related value expectation is $v i_{k} \leq v i_{k-} \mathrm{EV}$. When building the mathematical model under the conditions of maximum cost, it is necessary to assign $v i_{k-} \mathrm{EV}$ to $v i_{k}$, that is, $v i_{k}=v i_{k-} \mathrm{EV}$, where $v i_{k-} \mathrm{EV}$ is the maximum value of $v i_{k}$. Then, it is necessary to generate the optimisation configuration scheme for the GQPs to meet the other value expectations. This is a multiobjective optimisation problem with constraints. The linear weighting method is applied to convert it into a singleobjective optimisation problem. The physical meaning of the overall optimisation objective is that the fulfilment of value expectations except "Input Cost" is maximised as a whole with the support of the optimisation configuration scheme for the GQPs. Its mathematical model is given as follows:

$$
\max W=\sum_{i=1 \& i \neq k}^{m} v i_{-} w_{i}^{\prime \prime} \times P_{v i_{i-} \mathrm{EV}_{+}},
$$

where $v i \_w_{i}^{\prime}$ represents the importance ratings of the $i$ th optimisation objective $P_{v i_{i-} \mathrm{EV}}$, where $P_{v i_{i_{-}} \mathrm{EV}}$ is the $i$ th value expectation that should be met to the greatest extent and its calculation formula is

$$
P_{v i_{i-} \mathrm{EV}_{+}}=\frac{v i_{i-} \mathrm{EV}_{+}}{\sum_{j=1}^{n} \mu_{i j}^{*}+\mu_{i 0}^{*}},
$$

where $v i_{i-} \mathrm{EV}_{+}$represents the degree to which the realised value of the $i$ th VI is superior to limit value $v i_{i-} \mathrm{EV}$ of the $i$ th value expectation, that is, the degree to which the value expectation is met. Because some VIs are of positive type, the greater their value, the better their corresponding expectations are met, whereas others are of negative type, and hence, the smaller their value, the better their corresponding expectations are met. Thus, the calculation of $v i_{i-} \mathrm{EV}_{+}$is an absolute value:

$$
v i_{i-} \mathrm{EV}_{+}=\left|\sum_{j=1}^{n} \mu_{i j}^{*} g_{i j}\left(x_{j}\right)+\mu_{i 0}^{*}-v i_{i-} \mathrm{EV}\right| \text {. }
$$

Furthermore, because different VIs have different dimensions, it is necessary to normalise the degree to which the value expectation is met. $g_{i j}\left(x_{j}\right)$ represents the degree to which the change of the value of the $j$ th GQP affects the realisation of the $i$ th VIs, and its maximum value is one. When $g_{i j}\left(x_{j}\right)=1$, parameter $\mu_{i 0}^{*}+\sum_{j=1}^{n} \mu_{i j}^{*}$ in equation (18) represents the maximum value of the $i$ th VI to be realised. The objective function of the mathematical model under the condition of maximum cost is expressed as

$$
\max W=\sum_{i=1 \& i \neq k}^{m} v i_{-} w_{i}^{\prime} \cdot \frac{\left|\sum_{j=1}^{n} \mu_{i j}^{*} g_{i j}\left(q_{j}\right)+\mu_{i o}^{*}-v i_{i-} \mathrm{EV}\right|}{\sum_{j=1}^{n} \mu_{i j}^{*}+\mu_{i o}^{*}} .
$$

When building the mathematical model under the condition of minimum cost, the purpose of the corresponding 
objective function is to pursue the minimum input cost on the premise of the worst fulfilment of the value expectations. Thus, the objective function of the mathematical model under the condition of minimum cost is expressed as

$$
\max \left|\sum_{j=1}^{n} \mu_{k j}^{*} g_{k j}\left(q_{j}\right)+\mu_{k o}^{*}-v i_{k-} \mathrm{EV}\right| .
$$

In equations (18) and (19), because the optimisation configuration scheme for GQPs is constrained in the feasible region, when operator $S$ is " $>$ " or " $\geq$," $\mu_{i j}^{*}=\mu_{i j}^{*}$, and when operator $S$ is "<" or " $\leq$," $\mu_{i j}^{*}=\overline{\mu_{i j}^{*}}$.

The two optimal configuration schemes for GQPs can be obtained by solving equations (18) and (19), as shown in Figure 4. Both can meet the value expectations of the stakeholders for transboundary services, but the former is superior to the latter because of the different input costs. When the values of GQPs are between the optimal configuration schemes of the maximum cost and minimum cost, the value expectations can also be met, and the values of GQPs are an appropriate configuration scheme for GQPs. Thus, the two optimal configuration schemes are considered as the upper and lower bounds of the set of optimal configuration schemes for GQPs.

To summarise, by solving equations (18) and (19), the value ranges of GQPs under a certain confidence level can be obtained, that is, the set of optimisation configuration schemes for GQPs. If no feasible solution is found when solving the above model, this means that under the given constraints of GQPs, the lowest fulfilment of value expectations cannot be achieved. The designers can determine the configuration scheme for GQPs in which the VIs are realised to the maximal degree under the current constraint condition and can take it as a reference standard for the negotiated adjustment of value expectations by designers of transboundary services. Based on this configuration scheme, the designers need to decide whether to reduce the corresponding value expectations by conducting a negotiation among multiple participants or to identify potential partners who can provide higher service capabilities.

\section{Case Study}

In this study, we applied the proposed VQD to the GQP design of DiDi. Unlike traditional taxi services, DiDi is a transboundary service because it can provide higher service values for multiple stakeholders (e.g., DiDi app provider, map service provider, taxi drivers, and individual travellers) through deeply converging many independent services in different domains (e.g., traditional taxi services, mobile payment services, and map services). VQD can help the designers to make an appropriate decision, which results in a better configuration scheme for GQPs.

When designing DiDi, the designers should first communicate with the stakeholders to collect their respective value expectations, as shown in Table 3.

Next, we need to determine the relative importance ratings of the six VIs in Table 3. As described in Section 3.2, we adopt an FAHP; the detailed description of the FAHP is shown in Figure 5. Then, relative importance ratings VIW = $\left\{v i \_w_{1}, v i \_w_{2}, \ldots, v i \_w_{6}\right\}=\{0.211,0.183,0.139,0.124,0.145$, $0.198\}$ are obtained.

Next are the generation of the GQPs of DiDi and the identification of value constraints on the GQPs. The results are shown in Table 4.

Then, the designers need to identify the one-to-one basic type of quantitative mapping between each quality parameter $q_{j}$ and impact degree $v i_{i} d$ of each VI. Because of space limitations, only a partial set of influence degree relation functions is provided in Table 5.

Next, the designers need to provide the relevant data samples, as shown in Tables 6 and 7. Based on the given inputoutput sample sets, using the FLAR model, the objective function can be transformed into a linear programming model. The fuzzy coefficients that result from substituting the data into six linear programming models are shown in Table 8 .

In Table 8, row " $s$ " represents the spreads of the fuzzy numbers and row " $c$ " represents the centre point values of the fuzzy members. Next, we need to identify the correlation among GQPs. Similar to the previous method, after a series of operations and ignoring the spread value, we can derive a correlation between Maximum Waiting Time during Peak Period $q_{2}$ and Quantity of Available Taxis $q_{3}$. As $q_{3}$ changes, $q_{2}$ also changes. This correlation can be expressed as $q_{2}=-0.004 \times q_{3}+23.42$.

Using VIW and $\mu_{i j}^{*}$ obtained above, we can calculate the importance rating of the GQPs. Here, only the centre point values of the fuzzy numbers are considered. To overcome the influence of different dimensions, the centre point values of the parameters in Table 8 are normalised by column, and the relationship matrix of VIs versus GQPs is obtained as follows:

$$
P=\left(\begin{array}{llllll}
0.3194 & 0.2107 & 0.2267 & 0.1419 & 0.0772 & 0.0241 \\
0.2655 & 0.1388 & 0.3397 & 0.0962 & 0.1529 & 0.0069 \\
0.7174 & 0.0000 & 0.0000 & 0.1435 & 0.1391 & 0.0000 \\
0.0530 & 0.4022 & 0.0698 & 0.1184 & 0.2755 & 0.0811 \\
0.4022 & 0.2947 & 0.0000 & 0.1527 & 0.1504 & 0.0000 \\
0.3245 & 0.2130 & 0.0000 & 0.1404 & 0.1857 & 0.1364
\end{array}\right) .
$$

$P_{i j}$ represents the coefficient between the $i$ th VI and $j$ th GQP. The importance rating of the GQPs can be obtained by calculation as follows:

$$
\mathrm{QW}=\{0.3448,0.2046,0.1187,0.1321,0.1563,0.0434\} .
$$

Next, we need to generate an optimal configuration scheme for GQPs. The $\lambda$-cut set of the fuzzy number is obtained by substituting the data obtained above into the nonlinear programming model and setting confidence $\lambda$ to 0.2 , as shown in Table 9.

By substituting the upper and lower bounds of the $\lambda$-cut set into the nonlinear programming model, two nonlinear programming models that consist of precise parameters are obtained: 


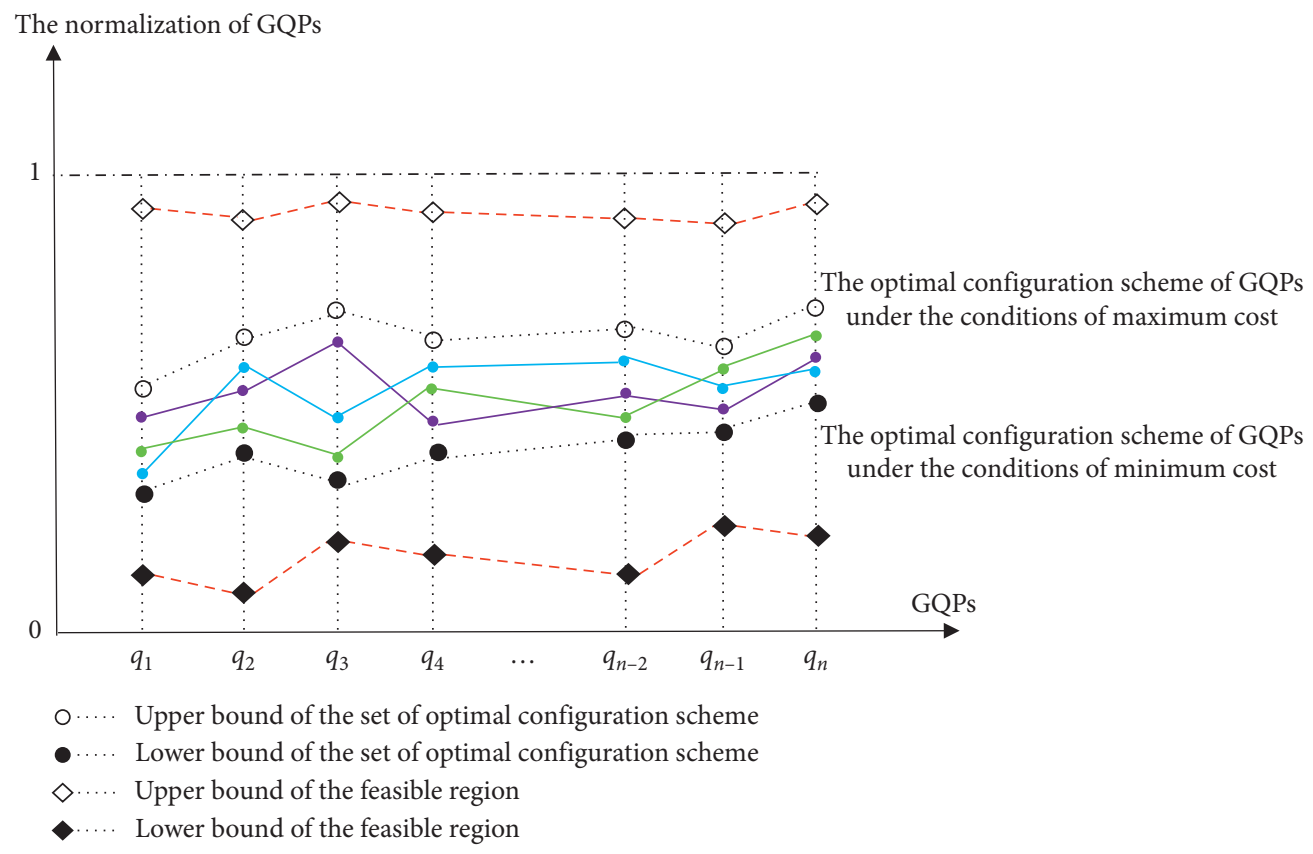

Figure 4: Set of optimal configuration schemes for GQPs.

TABle 3: Value expectations declared by stakeholders for DiDi.

\begin{tabular}{lcc}
\hline Value indicators & Value expectations (VEs) & Stakeholders related to VEs \\
\hline$v i_{1}:$ Market Share & $v p_{1}=\left(v i_{1}, 70 \%,>\right)$ & DiDi app provider \\
$v i_{2}:$ Daily Order Volume & $v p_{2}=\left(v i_{2}, 0.2\right.$ million, $\left.>\right)$ & PSP \\
$v i_{3}:$ Average Income per Order & $v p_{3}=\left(v i_{3}, 25\right.$ Yuan, $\left.>\right)$ & MSP \\
$v i_{4}:$ Operation Cost & $v p_{4}=\left(v i_{4}, 2.55\right.$ million Yuan, $\left.<\right)$ & DiDi app provider \\
$v i_{5}:$ Average Daily Income & $v p_{5}=\left(v i_{5}, 320\right.$ Yuan, $\left.>\right)$ & Taxi drivers \\
$v i_{6}:$ Favourable Rate & $v p_{6}=\left(v i_{6}, 50 \%,>\right)$ & Individual travellers \\
\hline
\end{tabular}

PSP: payment service provider; MSP: map service provider.

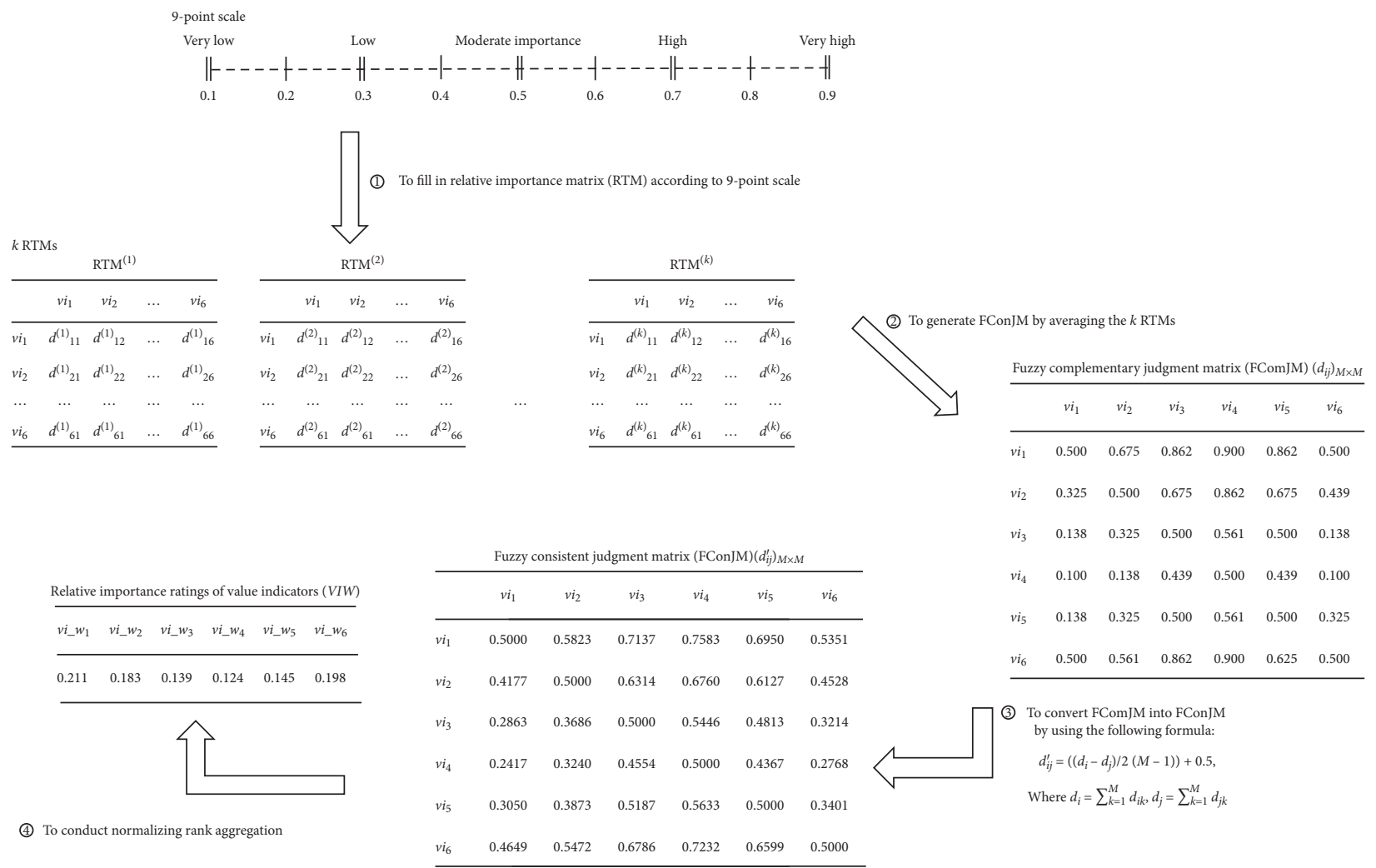

Figure 5: Detailed description of the FAHP. 
TABLE 4: GQPs and their value constraints.

\begin{tabular}{lc}
\hline GQPs & Value constraints on GQPs \\
\hline$q_{1}:$ Average Travel Cost & $c t q_{1}=[1.4 \mathrm{Yuan} / \mathrm{km}, 2.0 \mathrm{Yuan} / \mathrm{km}]$ \\
$q_{2}:$ Maximum Waiting Time during Peak Period & $c t q_{2}=[5 \mathrm{~min}, 20 \mathrm{~min}]$ \\
$q_{3}:$ Quantity of Available Taxis & $c t q_{3}=[3500$ vehicles, 5000 vehicles $]$ \\
$q_{4}:$ Drivers' Service Attitude & $c t q_{4}=\{1,2,3,4,5\}$ \\
$q_{5}:$ Security Guarantee Level & $c t q_{5}=\{1,2,3\}$ \\
$q_{6}:$ Positioning Accuracy & $c t q_{6}=\{10 \mathrm{~m}, 50 \mathrm{~m}\}$ \\
\hline
\end{tabular}

TABle 5: Partial influence degree relation functions.

\begin{tabular}{|c|c|c|}
\hline Functions & Expressions & Value range of independent variables \\
\hline$v i_{1} d=g_{11}\left(q_{1}\right)$ & $v i_{1} d=\left(e^{7} /\left(e^{7}-1\right)\right) \cdot e^{-7 \cdot\left(q_{1}-1.4 / 2.0-1.4\right)}-\left(1 /\left(e^{7}-1\right)\right)$ & $q_{1} \in[1.4,2.0]$ \\
\hline$v i_{1-} d=g_{12}\left(q_{2}\right)$ & $v i_{1} d=-\left(1 /\left(e^{6}-1\right)\right) \cdot e^{6 \cdot\left(q_{2}-5 / 20-5\right)}+\left(e^{6} /\left(e^{6}-1\right)\right)$ & $q_{2} \in[5,20]$ \\
\hline$v i_{1} \_d=g_{13}\left(q_{3}\right)$ & $v i_{1} d=(1 / 5000) \cdot q_{3}$ & $q_{3} \in[3500,5000]$ \\
\hline$v i_{1-} d=g_{14}\left(q_{4}\right)$ & $v i_{1-} d=\left\{\begin{array}{l}0, q_{4}=1 \\
0.25, q_{4}=2 \\
0.5, q_{4}=3 \\
0.75, q_{4}=4 \\
1, q_{4}=5\end{array}\right.$ & $q_{4} \in[1,2,3,4,5]$ \\
\hline$v i_{1} d=g_{15}\left(q_{5}\right)$ & $v i_{1} \_d=\left\{\begin{array}{l}0, q_{5}=1 \\
0.7, q_{5}=2 \\
1, q_{5}=3\end{array}\right.$ & $q_{5} \in[1,2,3]$ \\
\hline$v i_{1} d=g_{16}\left(q_{6}\right)$ & $v i_{1} \_d=(1 / 5000) \cdot q_{3}$ & $q_{6} \in[10,50]$ \\
\hline
\end{tabular}

TABLE 6: GQPs in sample sets.

\begin{tabular}{|c|c|c|c|c|c|c|}
\hline & $q_{1}$ & $q_{2}$ & $q_{3}$ & $q_{4}$ & $q_{5}$ & $q_{6}$ \\
\hline Sample 1 & 1.5 & 7 & 4000 & 5 & 2 & 10 \\
\hline Sample 2 & 2.0 & 18 & 1500 & 4 & 3 & 15 \\
\hline Sample 3 & 1.5 & 9 & 3500 & 4 & 1 & 20 \\
\hline Sample 4 & 1.7 & 12 & 2500 & 3 & 1 & 30 \\
\hline Sample 5 & 1.6 & 6 & 4500 & 5 & 3 & 15 \\
\hline Sample 6 & 2.0 & 10 & 3000 & 2 & 1 & 25 \\
\hline Sample 7 & 1.5 & 11 & 3200 & 3 & 2 & 30 \\
\hline Sample 8 & 1.9 & 15 & 2000 & 4 & 2 & 15 \\
\hline Sample 9 & 1.7 & 12 & 3200 & 3 & 1 & 20 \\
\hline Sample 10 & 1.5 & 11 & 3200 & 3 & 2 & 20 \\
\hline Sample 11 & 1.8 & 18 & 1500 & 4 & 3 & 25 \\
\hline Sample 12 & 1.5 & 9 & 3500 & 3 & 2 & 30 \\
\hline
\end{tabular}

TABLE 7: Value expectations in sample sets.

\begin{tabular}{|c|c|c|c|c|c|c|}
\hline & $p v i_{1}^{*}$ & $p v i_{2}^{*}$ & $p v i_{3}^{*}$ & $p v i_{4}^{*}$ & $p v i_{5}^{*}$ & $p v i_{6}^{*}$ \\
\hline Sample 1 & $(0.91,0.03)$ & $(28.5,1)$ & $(25,1)$ & $(275,5)$ & $(340,5)$ & $(0.77,0.02)$ \\
\hline Sample 2 & $(0.45,0.02)$ & $(17.5,0.2)$ & $(32,2)$ & $(165,5)$ & $(325,5)$ & $(0.4,0.02)$ \\
\hline Sample 3 & $(0.75,0.03)$ & $(20.5,0.5)$ & $(21,1)$ & $(205,5)$ & $(280,5)$ & $(0.43,0.02)$ \\
\hline Sample 4 & $(0.53,0.03)$ & $(12.8,0.5)$ & $(22,2)$ & $(145,5)$ & $(300,5)$ & $(0.18,0.02)$ \\
\hline Sample 5 & $(0.88,0.03)$ & $(29,0.5)$ & $(28,1)$ & $(320,5)$ & $(390,5)$ & $(0.7,0.02)$ \\
\hline Sample 6 & $(0.5,0.03)$ & $(12.5,0.3)$ & $(24.5,2)$ & $(165,5)$ & $(292,5)$ & $(0.14,0.02)$ \\
\hline Sample 7 & $(0.75,0.03)$ & $(22,0.5)$ & $(23,1)$ & $(200,5)$ & $(305,5)$ & $(0.45,0.02)$ \\
\hline Sample 8 & $(0.6,0.03)$ & $(15,0.5)$ & $(29,2)$ & $(165,5)$ & $(345,5)$ & $(0.36,0.02)$ \\
\hline Sample 9 & $(0.62,0.02)$ & $(14.5,0.5)$ & $(22,2)$ & $(175,5)$ & $(300,5)$ & $(0.2,0.02)$ \\
\hline Sample 10 & $(0.77,0.02)$ & $(22,0.5)$ & $(23,1)$ & $(215,5)$ & $(305,5)$ & $(0.5,0.02)$ \\
\hline Sample 11 & $(0.5,0.02)$ & $(15,0.2)$ & $(29,2)$ & $(160,5)$ & $(325,5)$ & $(0.35,0.02)$ \\
\hline Sample 12 & $(0.78,0.02)$ & $(25,0.5)$ & $(23,1)$ & $(220,5)$ & $(305,5)$ & $(0.5,0.02)$ \\
\hline
\end{tabular}


TABle 8: Fuzzy coefficients.

\begin{tabular}{|c|c|c|c|c|c|c|c|}
\hline \multicolumn{2}{|c|}{$\mu_{i j}^{*}$} & $i=1$ & $i=2$ & $i=3$ & $i=4$ & $i=5$ & $i=6$ \\
\hline \multirow{2}{*}{$j=0$} & c & 0.0000 & 3.3319 & 0.0000 & 0.0000 & 0.0000 & 0.0000 \\
\hline & $\mathrm{s}$ & 0.0076 & 0.0914 & 0.0000 & 5.0000 & 5.0000 & 0.0053 \\
\hline \multirow{2}{*}{$j=1$} & c & 0.4090 & 9.3727 & 23.3333 & 0.5828 & 161.4414 & 0.3521 \\
\hline & $\mathrm{s}$ & 0.0000 & 0.3658 & 2.0000 & 0.0000 & 0.0000 & 0.0000 \\
\hline \multirow{2}{*}{$j=2$} & c & 0.2698 & 4.9006 & 0.0000 & 4.4202 & 118.3024 & 0.2310 \\
\hline & $\mathrm{s}$ & 0.0225 & 0.0000 & 0.0000 & 0.0000 & 0.0000 & 0.0125 \\
\hline \multirow{2}{*}{$j=3$} & c & 0.2903 & 11.9931 & 0.0000 & 0.7669 & 0.0000 & 0.0000 \\
\hline & $\mathrm{s}$ & 0.0000 & 0.3448 & 0.0000 & 0.0000 & 0.0000 & 0.0000 \\
\hline \multirow{2}{*}{$j=4$} & c & 0.1817 & 3.3942 & 4.6666 & 1.3006 & 61.2664 & 0.1523 \\
\hline & $\mathrm{s}$ & 0.0000 & 0.0069 & 0.0000 & 0.0000 & 0.0000 & 0.0000 \\
\hline \multirow{2}{*}{$j=5$} & c & 0.0988 & 5.3985 & 4.5238 & 3.0279 & 60.3799 & 0.2014 \\
\hline & $\mathrm{s}$ & 0.0000 & 0.0000 & 0.0000 & 0.0000 & 0.0000 & 0.0000 \\
\hline \multirow{2}{*}{$j=6$} & c & 0.0308 & 0.2407 & 0.0000 & 0.8908 & 0.0000 & 0.1479 \\
\hline & $\mathrm{s}$ & 0.0000 & 0.0000 & 0.0000 & 0.0000 & 0.0000 & 0.0000 \\
\hline
\end{tabular}

TABle 9: Confidence interval (CI) corresponding to the fuzzy coefficient.

\begin{tabular}{|c|c|c|c|c|c|c|c|}
\hline \multicolumn{2}{|c|}{ CI of $\mu_{i j}^{*}$} & $i=1$ & $i=2$ & $i=3$ & $i=4$ & $i=5$ & $i=6$ \\
\hline \multirow{2}{*}{$j=0$} & $\mathrm{lb}$ & -0.00608 & 3.25878 & 0.0000 & -4.0000 & -4.0000 & -0.00424 \\
\hline & $\mathrm{ub}$ & 0.00608 & 3.40502 & 0.0000 & 4.0000 & 4.0000 & 0.00424 \\
\hline \multirow{2}{*}{$j=1$} & $\mathrm{lb}$ & 0.4090 & 9.08006 & 21.7333 & 0.5828 & 161.4414 & 0.3521 \\
\hline & $\mathrm{ub}$ & 0.4090 & 9.66534 & 24.9333 & 0.5828 & 161.4414 & 0.3521 \\
\hline \multirow{2}{*}{$j=2$} & $\mathrm{lb}$ & 0.2518 & 4.9006 & 0.0000 & 4.4202 & 118.3024 & 0.2210 \\
\hline & $\mathrm{ub}$ & 0.2878 & 4.9006 & 0.0000 & 4.4202 & 118.3024 & 0.2410 \\
\hline \multirow{2}{*}{$j=3$} & $\mathrm{lb}$ & 0.2903 & 11.7173 & 0.0000 & 0.7669 & 0.0000 & 0.0000 \\
\hline & $\mathrm{ub}$ & 0.2903 & 12.2690 & 0.0000 & 0.7669 & 0.0000 & 0.0000 \\
\hline \multirow{2}{*}{$j=4$} & $\mathrm{lb}$ & 0.1817 & 3.3887 & 4.6666 & 1.3006 & 61.2664 & 0.1523 \\
\hline & $\mathrm{ub}$ & 0.1817 & 3.3997 & 4.6666 & 1.3006 & 61.2664 & 0.1523 \\
\hline \multirow{2}{*}{$j=5$} & $\mathrm{lb}$ & 0.0988 & 5.3985 & 4.5238 & 3.0279 & 60.3799 & 0.2014 \\
\hline & $\mathrm{ub}$ & 0.0988 & 5.3985 & 4.5238 & 3.0279 & 60.3799 & 0.2014 \\
\hline \multirow{2}{*}{$j=6$} & $\mathrm{lb}$ & 0.0308 & 0.2407 & 0.0000 & 0.8908 & 0.0000 & 0.1479 \\
\hline & $\mathrm{ub}$ & 0.0308 & 0.2407 & 0.0000 & 0.8908 & 0.0000 & 0.1479 \\
\hline
\end{tabular}

CI: the confidence interval; lb: the lower bound of the confidence interval; ub: the upper bound of the confidence interval.

$$
\begin{aligned}
& \max W=\sum_{i=1 \& i \neq 4}^{6} v i \_w_{i}^{\prime} \cdot \frac{\left|\sum_{j=1}^{6} \mu_{i j} g_{i j}\left(q_{j}\right)+\mu_{i 0}-v i_{i-} \mathrm{EV}\right|}{\sum_{j=1}^{6} \mu_{i j}+\mu_{i 0}}, \\
& \text { s.t. }\left\{\begin{array}{l}
\sum_{j=1}^{6} \mu_{1 j} g_{1 j}\left(q_{j}\right)+\mu_{10} \geq 0.7, \sum_{j=1}^{6} \mu_{2 j} g_{2 j}\left(q_{j}\right)+\mu_{20} \geq 20, \sum_{j=1}^{6} \mu_{3 j} g_{3 j}\left(q_{j}\right)+\mu_{30} \geq 25, \\
\sum_{j=1}^{6} \mu_{4 j} g_{4 j}\left(q_{j}\right)+\mu_{40} \leq 300, \sum_{j=1}^{6} \mu_{5 j} g_{5 j}\left(q_{j}\right)+\mu_{50} \geq 320, \sum_{j=1}^{6} \mu_{6 j} g_{6 j}\left(q_{j}\right)+\mu_{60} \geq 0.5, \\
q_{2}=-0.004 q_{3}+23.42, \\
1.4 \leq q_{1} \leq 2.0,5 \leq q_{2} \leq 20,3500 \leq q_{3} \leq 5000,10 \leq q_{6} \leq 50, \\
q_{3} \in[1,2,3,4,5], \\
q_{4} \in[1,2,3],
\end{array}\right.
\end{aligned}
$$




$$
\begin{aligned}
& \min W=\left|\sum_{j=1}^{6} \mu_{4 j} g_{4 j}\left(q_{j}\right)+\mu_{40}-300\right|, \\
& \text { s.t. }\left\{\begin{array}{l}
\sum_{j=1}^{6} \mu_{1 j} g_{1 j}\left(q_{j}\right)+\mu_{10} \geq 0.7, \sum_{j=1}^{6} \mu_{2 j} g_{2 j}\left(q_{j}\right)+\mu_{20} \geq 20, \sum_{j=1}^{6} \mu_{3 j} g_{3 j}\left(q_{j}\right)+\mu_{30} \geq 25, \\
\sum_{j=1}^{6} \mu_{4 j} g_{4 j}\left(q_{j}\right)+\mu_{40} \leq 300, \sum_{j=1}^{6} \mu_{5 j} g_{5 j}\left(q_{j}\right)+\mu_{50} \geq 320, \sum_{j=1}^{6} \mu_{6 j} g_{6 j}\left(q_{j}\right)+\mu_{60} \geq 0.5, \\
q_{2}=-0.004 q_{3}+23.42, \\
1.4 \leq q_{1} \leq 2.0,5 \leq q_{2} \leq 20,3500 \leq q_{3} \leq 5000,10 \leq q_{6} \leq 50, \\
q_{3} \in[1,2,3,4,5], \\
q_{4} \in[1,2,3] .
\end{array}\right.
\end{aligned}
$$

By solving equations (22) and (23), two optimal configuration schemes $Q_{1}=(1.5,8.8,3647.5,5,3,50)$ and $Q_{2}=(1.6,9.4,3500,5,3,50)$ can be obtained. Therefore, the set of optimal configuration schemes for GQPs for DiDi is as follows:

(i) $q_{1}$-Average Travel Cost (Yuan $\left./ \mathrm{km}\right):[1.5,1.6]$

(ii) $q_{2}$-Maximum Waiting Time during Peak Period (min): $[8.8,9.4]$

(iii) $q_{3}$-Quantify of Available Taxis (vehicle): [3500, 3648]

(iv) $q_{4}$-Driver's Service Attitude (1 5): $[5,5]$

(v) $q_{5}$-Security Guarantee Level $(1 \sim 3):[3,3]$

(vi) $q_{6}$-Positioning Accuracy $(\mathrm{m}):[50,50]$

In the above case, DiDi is in the initial stage of operation, and its business goal is to open up the market. At this time, its business strategy is to increase the cost of investment to provide high-value and high-quality services at a more favourable price to attract more individual travellers and gain more market share. From the above set of optimal configuration schemes for GQPs, we know that the value range of "Average Travel Cost" is $[1.5,1.6]$, which means that DiDi's price is relatively favourable and the value range of "Maximum Waiting Time during Peak Period" is $[8.8,9.4]$, which means that the individual traveller's waiting time is relatively short. The value range of "Driver's Service Attitude" is $[5,5]$ and the value range of "Security Guarantee Level" is [3,3]. The values of these two quality parameters have reached their respective maximum values, and they all have a greater impact on "Market Share." By contrast, the value range of "Quantify of Available Taxis" is [3500, 3648] and the value range of "Positioning Accuracy" is [50, 50]. The value range of these two GQPs is not ideal, but their influence on "Market Share" is small. Overall, the configuration scheme for the above GQPs is in line with the design requirements of transboundary services.

\section{Conclusion and Future Work}

Based on QFD, we proposed a two-phase model called VQDQCD to facilitate designers to precisely design quality/capability characteristics of transboundary services by elaborating on the considerations of value expectation conflicts, quality attribute correlations, and constraints of the actual service capabilities of different service providers from different business domains. VQD-QCD can be applied to seek a trade-off between high-level value expectations and realworld service capabilities, and then the optimal configuration scheme for GQPs, LQPs, and CPs can be obtained in a top-down manner. In particular, considering the inherent vagueness and uncertainty of relationships among the VIs and GQPs of transboundary services, FLAR and fuzzy nonlinear programming methods were incorporated into VQD to identify quantitative relations between VIs and GQPs, and correlations among GQPs.

There are two issues that still need to be solved in our VQD-QCD. One is the lack of related data. The other is that because of the lack of related data, there has been no in-depth study on negotiation design for the bottom-up approach. For the former, more data related to models in VQD can be collected during the execution of the designed transboundary service. In this case, the parameters of these models would be continuously optimised and then the updated VQD could be used to obtain a more appropriate configuration scheme for GQPs. For the latter, this is future work because when the available capabilities of stakeholders in the real-world service cannot meet their value expectations, this bottom-up negotiation method can help multiple stakeholders to make reasonable and iterative adjustments to their respective value expectations. The adjustment scheme of the value expectations can help to reconduct the generative design and obtain a reasonable configuration scheme. Therefore, future work will be to conduct an in-depth study on this negotiation design method based on game theory to further improve VQD and then help designers to achieve the GQP design of transboundary services.

\section{Data Availability}

No data were used to support this study.

\section{Conflicts of Interest}

The authors declare that they have no conflicts of interest. 


\section{Acknowledgments}

This work was partially supported by the National Key Research and Development Program of China (grant number 2017YFB1400604), the National Science Foundation of China (grant numbers 61772155, 61832014, 61832004, and 61802089), and the Natural Science Foundation of Heilongjiang Province, China (grant number QC2018081).

\section{References}

[1] Z. Wu, J. Yin, S. Deng, J. Wu, Y. Li, and L. Chen, "Modern service industry and crossover services: development and trends in China. modern service industry and transboundary services: development and trends in China," IEEE Transactions on Services Computing, vol. 9, no. 5, pp. 664-671, 2016.

[2] X. Xu, G. Motta, Z. Tu, H. Xu, Z. Xu, and X. Wang, "A new paradigm of software service engineering in big data and big service era," Computing, vol. 100, no. 4, pp. 353-368, 2018.

[3] Community Research and Development Information Service and European Commission, Towards the Internet of Services 2014, http://cordis.europa.eu/fp7/ict/ssai/.

[4] L.-K. Chan and M.-L. Wu, "Quality function deployment: a literature review," European Journal of Operational Research, vol. 143, no. 3, pp. 463-497, 2002.

[5] L. P. Sullivan, "Quality function deployment," Quality Progress, vol. 19, no. 6, pp. 39-50, 1986.

[6] L. R. Guinta and N. C. Praizler, The QFD Book: The Team Approach to Solving Problems and Satisfying Customers through Quality Function Deployment, Amacom, New York, NY, USA, 1993.

[7] A. Griffin and J. R. Hauser, "The voice of the customer," Marketing Science, vol. 12, no. 1, pp. 1-27, 1993.

[8] American Supplier Institute, Quality Function Deployment (Service QFD): 3-day Workshop, ASI Press, Dearborn, MI, USA, 1994.

[9] L. Cohen, Quality Function Deployment: How to Make QFD Work for You, Addison-Wesley, Reading, MA, USA, 1995.

[10] L. Chan and M. L. Wu, "A systematic approach to quality function deployment with a full illustrative example," Omega, vol. 33, no. 2, pp. 119-139, 2005.

[11] J. J. Hong, W. K. Chang, and H. K. Bo, "Requirements development process for manufacturing information systems based on quality function deployment," International Journal of Computer Integrated Manufacturing, vol. 31, no. 2, pp. 164-174, 2018.

[12] S. Liu, X. F. Xu, and Z. J. Wang, “A SQFD approach for service system design evaluation \& optimization," in Proceedings of the 5th International Conference on Interoperability for Enterprise Software and Applications, pp. 23-27, Beijing, China, April 2009.

[13] W. Zeng, Q. Feng, and J. Li, "Fuzzy least absolute linear regression," Applied Soft Computing, vol. 52, pp. 1009-1019, 2017.

[14] H.-J. Park, J.-S. Lim, and J. M. Kang, "Optimization of gas production systems using fuzzy nonlinear programming and co-evolutionary genetic algorithm," Energy Sources, Part A: Recovery, Utilization, and Environmental Effects, vol. 30, no. 9, pp. 818-825, 2008.

[15] Z. Xu and H. Liao, "Intuitionistic fuzzy analytic hierarchy process," IEEE Transactions on Fuzzy Systems, vol. 22, no. 4, pp. 749-761, 2014.
[16] Y. Xu, Q. Da, and L. Liu, "Normalizing rank aggregation method for priority of a fuzzy preference relation and its effectiveness," International Journal of Approximate Reasoning, vol. 50, no. 8, pp. 1287-1297, 2009.

[17] L. K. Chan, H. P. Kao, M. L. Wu, and M. L. Wu, "Rating the importance of customer needs in quality function deployment by fuzzy and entropy methods," International Journal of Production Research, vol. 37, no. 11, pp. 2499-2518, 1999.

[18] A. K. Sangaiah, D. V. Medhane, T. Han, M. S. Hossain, and G. Muhammad, "Enforcing position-based confidentiality with machine learning paradigm through mobile edge computing in real-time industrial informatics," IEEE Transactions on Industrial Informatics, vol. 15, no. 7, pp. 4189-4196, 2019.

[19] Y. Z. Chen and L. Chen, "A non-linear possibilistic regression approach to model functional relationships in product planning," International Journal of Advanced Manufacturing Technology, vol. 28, no. 11-12, pp. 1175-1181, 2006.

[20] S. M. Choi and J. J. Buckley, "Fuzzy regression using least absolute deviation estimators," Soft Computing, vol. 12, no. 3 , pp. 257-263, 2008.

[21] A. K. Sangaiah, G. Bian, S. M. Bozorgi, M. Y. Suraki, A. A. R. Hosseinabadi, and M. B. Shareh, "A novel quality-ofservice-aware web services composition using biogeographybased optimization algorithm," Soft Computing, 2019.

[22] S. Kaur, S. Singh, S. Kaushal, and A. K. Sangaiah, "Comparative analysis of quality metrics for community detection in social networks using genetic algorithm," Neural Network World, vol. 26, no. 6, pp. 625-641, 2016. 